\title{
Optimal matroid bases with intersection constraints: Valuated matroids, M-convex functions, and their applications*
}

\author{
Yuni Iwamasa ${ }^{\dagger} \quad$ Kenjiro Takazawa ${ }^{\ddagger}$
}

March 1, 2021

\begin{abstract}
For two matroids $M_{1}$ and $M_{2}$ with the same ground set $V$ and two cost functions $w_{1}$ and $w_{2}$ on $2^{V}$, we consider the problem of finding bases $X_{1}$ of $M_{1}$ and $X_{2}$ of $M_{2}$ minimizing $w_{1}\left(X_{1}\right)+w_{2}\left(X_{2}\right)$ subject to a certain cardinality constraint on their intersection $X_{1} \cap X_{2}$. For this problem, Lendl, Peis, and Timmermans (2019) discussed modular cost functions: they reduced the problem to weighted matroid intersection for the case where the cardinality constraint is $\left|X_{1} \cap X_{2}\right| \leq k$ or $\left|X_{1} \cap X_{2}\right| \geq k$; and designed a new primal-dual algorithm for the case where the constraint is $\left|X_{1} \cap X_{2}\right|=k$.

The aim of this paper is to generalize the problems to have nonlinear convex cost functions, and to comprehend them from the viewpoint of discrete convex analysis. We prove that each generalized problem can be solved via valuated independent assignment, valuated matroid intersection, or M-convex submodular flow, to offer a comprehensive understanding of weighted matroid intersection with intersection constraints. We also show the NP-hardness of some variants of these problems, which clarifies the coverage of discrete convex analysis for those problems. Finally, we present applications of our generalized problems in the recoverable robust matroid basis problem, combinatorial optimization problems with interaction costs, and matroid congestion games.
\end{abstract}

Keywords: Valuated independent assignment, valuated matroid intersection, Mconvex submodular flow, recoverable robust matroid basis problem, combinatorial optimization problem with interaction costs, congestion game

\section{Introduction}

Weighted matroid intersection is one of the most fundamental combinatorial optimization problems solvable in polynomial time. This problem generalizes a number of tractable problems including the maximum-weight bipartite matching and minimum-weight arborescence problems. The comprehension of mathematical structures of weighted matroid intersection, e.g., Edmonds' intersection theorem [7] and combinatorial primal-dual algorithm [9, 16, 20], contributes to the development of algorithmics in combinatorial optimization as well as matroid theory.

In this article, we represent a matroid by a pair of its ground finite set $V$ and base family $\mathcal{B} \subseteq 2^{V}$, which satisfy the following exchange axiom: for $X, Y \in \mathcal{B}$ and $v \in X \backslash Y$, there exists

\footnotetext{
${ }^{*}$ A preliminary version of this paper appears in the proceedings of the 16th Annual Conference on Theory and Applications of Models of Computation (TAMC 2020). This work was done while Yuni Iwamasa was at National Institute of Informatics.

${ }^{\dagger}$ Department of Communications and Computer Engineering, Graduate School of Informatics, Kyoto University, Kyoto 606-8501, Japan. Email: iwamasa@i.kyoto-u.ac.jp

${ }^{\ddagger}$ Department of Industrial and Systems Engineering, Faculty of Science and Engineering, Hosei University, Tokyo 184-8584, Japan. Email: takazawa@hosei.ac.jp
} 
$u \in Y \backslash X$ such that $X \backslash\{v\} \cup\{u\} \in \mathcal{B}$. For a base family $\mathcal{B}$, the set family $\mathcal{I}:=\{I \subseteq B \mid B \in \mathcal{B}\}$ is called the independent set family. It is well known (see e.g., [36]) that the base family uniquely determines the corresponding independent set family, and vice versa. Hence in this paper we also represent a matroid by the pair $(V, \mathcal{I})$ of the ground set $V$ and the independent set family $\mathcal{I}$.

Let $M_{1}=\left(V, \mathcal{B}_{1}\right)$ and $M_{2}=\left(V, \mathcal{B}_{2}\right)$ be matroids on $V$ with base families $\mathcal{B}_{1}$ and $\mathcal{B}_{2}$, respectively. Also let $w_{1}$ and $w_{2}$ be weight functions on $V$ and $k$ a nonnegative integer. A weight function $w: V \rightarrow \mathbf{R}$ is also regarded as a modular function $w: 2^{V} \rightarrow \mathbf{R}$ defined by $w(X)=\sum_{v \in X} w(v)$ for each $X \subseteq V$. Recently, Lendl, Peis, and Timmermans [24] have introduced the following variants of weighted matroid intersection, in which a cardinality constraint is imposed on the intersection:

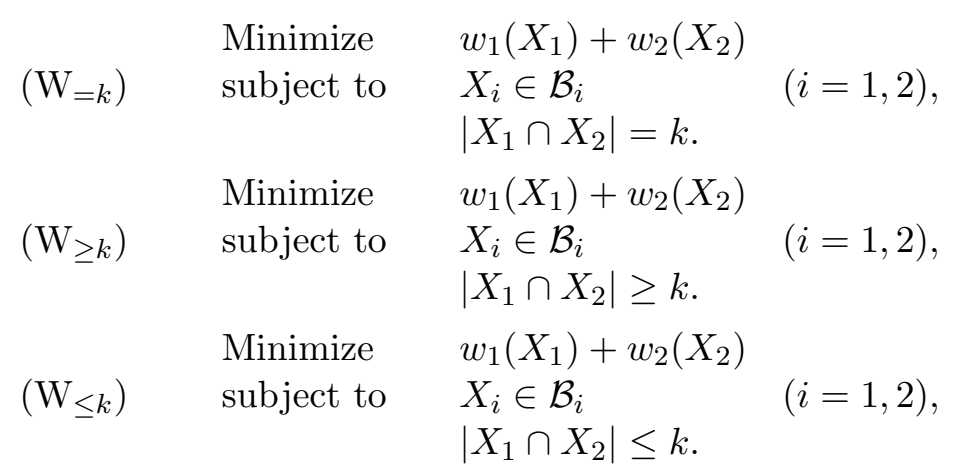

We remark here that the tractability of $\left(\mathrm{W}_{=k}\right)$ implies that of $\left(\mathrm{W}_{\geq k}\right)$ and $\left(\mathrm{W}_{\leq k}\right)$. Indeed, for example, we obtain an optimal solution for $\left(\mathrm{W}_{\geq k}\right)$ for $k=\ell$ by solving $\left(\mathrm{W}_{=k}\right)$ for $k=$ $\ell, \ell+1, \ldots,|V|$ and returning a minimum solution over them.

The motivation of these problems comes from the recoverable robust matroid basis problem [3]. Lendl et al. [24] showed that $\left(\mathrm{W}_{=k}\right),\left(\mathrm{W}_{\geq k}\right)$, and $\left(\mathrm{W}_{\leq k}\right)$ are strongly polynomial-time solvable: they developed a new primal-dual algorithm for $\left(\mathrm{W}_{=k}\right)$; and reduced $\left(\mathrm{W}_{\geq k}\right)$ and $\left(\mathrm{W}_{\leq k}\right)$ to weighted matroid intersection. By this result, they affirmatively settled an open question on the strongly polynomial-time solvability of the recoverable robust matroid basis problem under interval uncertainty representation $[14,15]$.

Lendl et al. [24] further discussed two kinds of generalizations of the above problems. One is to consider more than two matroids. Let $n$ be a positive integer, and $[n]:=\{1,2, \ldots, n\}$. For each $i \in[n]$, let $M_{i}=\left(V, \mathcal{B}_{i}\right)$ be a matroid with ground set $V$ and base family of $\mathcal{B}_{i}$. For instance, $\left(\mathrm{W}_{\leq k}\right)$ can be generalized as follows.

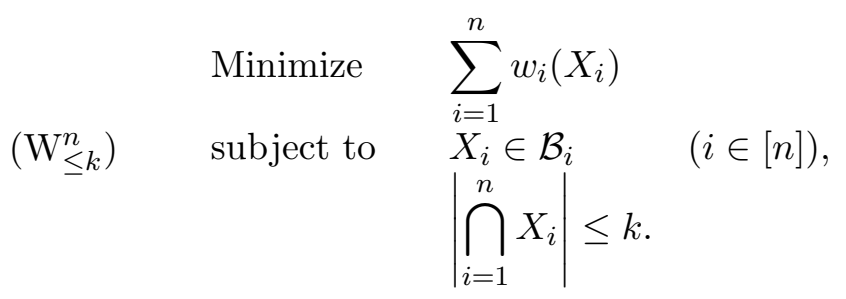

Generalizations of $\left(\mathrm{W}_{=k}\right)$ and $\left(\mathrm{W}_{\geq k}\right)$, which we name $\left(\mathrm{W}_{=k}^{n}\right)$ and $\left(\mathrm{W}_{\geq k}^{n}\right)$, respectively, can be obtained in the same way. Lendl et al. [24] proved that $\left(\mathrm{W}_{-k}^{n}\right)$ and $\left(\mathrm{W}_{\geq k}^{n}\right)$ are NP-hard, whereas $\left(\mathrm{W}_{\leq k}^{n}\right)$ can be solved in strongly polynomial time. Indeed, they reduced $\left(\mathrm{W}_{\leq k}^{n}\right)$ to weighted matroid intersection.

The other is a polymatroidal generalization. Let $B_{1}, B_{2} \subseteq \mathbf{Z}^{V}$ be the base polytopes of some polymatroids on the ground set $V$. The following problem generalizes $\left(\mathrm{W}_{\geq k}\right)$, where $w_{1}$ and $w_{2}$ 
are linear functions on $\mathbf{Z}^{V}$.

$$
\begin{array}{lll} 
& \text { Minimize } & w_{1}\left(x_{1}\right)+w_{2}\left(x_{2}\right) \\
& \text { subject to } & x_{i} \in B_{i}
\end{array} \quad(i=1,2),
$$

Again, generalizations of $\left(\mathrm{W}_{=k}\right)$ and $\left(\mathrm{W}_{\leq k}\right)$ can be obtained in the same manner, and they are NP-hard. Lendl et al. [24] proved that $\left(\mathrm{P}_{\geq k}\right)$ can be reduced to the polymatroidal flow problem [13, 21, 22], which is equivalent to the submodular flow problem [8] (see [11]), and thus can be solved in strongly polynomial time.

The aim of this paper is to offer a comprehensive understanding of the above problems in view of discrete convex analysis (DCA) [29,32], particularly focusing on $M$-convexity [26]. DCA provides a theory of convex functions on the integer lattice $\mathbf{Z}^{V}$. M-convex functions play central roles in DCA and naturally appear in various research fields such as combinatorial optimization, economics, and game theory [33, 34].

M-convex functions are a quantitative generalization of matroids. The formal definition of M-convex functions is given as follows. A function $f: \mathbf{Z}^{V} \rightarrow \mathbf{R} \cup\{+\infty\}$ is said to be $M$-convex if it satisfies the following generalization of the matroid exchange axiom: for all $x=(x(v))_{v \in V}$ and $y=(y(v))_{v \in V}$ with $x, y \in \operatorname{dom} f$, and all $v \in V$ with $x(v)>y(v)$, there exists $u \in V$ with $x(u)<y(u)$ such that

$$
f(x)+f(y) \geq f\left(x-\chi_{v}+\chi_{u}\right)+f\left(y+\chi_{v}-\chi_{u}\right),
$$

where dom $f$ denotes the effective domain $\left\{x \in \mathbf{Z}^{V} \mid f(x)<+\infty\right\}$ of $f$ and $\chi_{v}$ the $v$-th unit vector for $v \in V$. In particular, if $\operatorname{dom} f$ is included in the hypercube $\{0,1\}^{V}$, then $f$ is called a valuated matroid ${ }^{1}[5,6]$.

In this paper, we address the following M-convex (and hence nonlinear) generalizations of $\left(\mathrm{W}_{=k}\right),\left(\mathrm{W}_{\geq k}\right),\left(\mathrm{W}_{\leq k}^{n}\right)$, and $\left(\mathrm{P}_{\geq k}\right)$, and present their applications. Let $\omega_{1}, \omega_{2}, \ldots, \omega_{n}$ be valuated matroids on $2^{V}$, where we identify $2^{V}$ with $\{0,1\}^{V}$ by the natural correspondence between $X \subseteq V$ and $x \in\{0,1\}^{V} ; x(v)=1$ if and only if $v \in X$.

- For $\left(\mathrm{W}_{=k}\right)$ and $\left(\mathrm{W}_{\geq k}\right)$, by generalizing the weight functions $w_{1}$ and $w_{2}$ to valuated matroids $\omega_{1}$ and $\omega_{2}$, we obtain:

$$
\begin{array}{lll}
\left(\mathrm{V}_{=k}\right) & \begin{array}{l}
\text { Minimize } \\
\text { subject to }
\end{array} & \left.\omega_{1}\left(X_{1}\right)+\omega_{1} \cap X_{2}\right) \\
& \text { Minimize } & \omega_{1}\left(X_{1}\right)+\omega_{2}\left(X_{2}\right) \\
\left(\mathrm{V}_{\geq k}\right) & \begin{array}{l}
\text { subject to } \\
\text { subjoct }
\end{array} & \left|X_{1} \cap X_{2}\right| \geq k .
\end{array}
$$

Again observe that the tractability of $\left(\mathrm{V}_{=k}\right)$ implies that of $\left(\mathrm{V}_{\geq k}\right)$.

- For $\left(\mathrm{W}_{<k}^{n}\right)$ (and hence $\left(\mathrm{W}_{\leq k}\right)$ as well), in addition to generalizing $w_{1}, w_{2}, \ldots, w_{n}$ to valuated matroids $\omega_{1}, \omega_{2}, \ldots, \omega_{n}$, we generalize the cardinality constraint $\left|\bigcap_{i=1}^{n} X_{i}\right| \leq k$ to a matroid constraint. Namely, let $M=(V, \mathcal{I})$ be a new matroid, where $\mathcal{I}$ denotes its independent set family, and generalize $\left(\mathrm{W}_{\leq k}^{n}\right)$ as follows.

$\left(\mathrm{V}_{\mathcal{I}}^{n}\right)$

$$
\begin{array}{ll}
\text { Minimize } & \sum_{i=1}^{n} \omega_{i}\left(X_{i}\right) \\
\text { subject to } & \bigcap_{i=1}^{n} X_{i} \in \mathcal{I} .
\end{array}
$$

\footnotetext{
${ }^{1}$ The original definition of a valuated matroid is an $M$-concave function, i.e., the negation of an M-convex function, whose effective domain is included in the hypercube.
} 


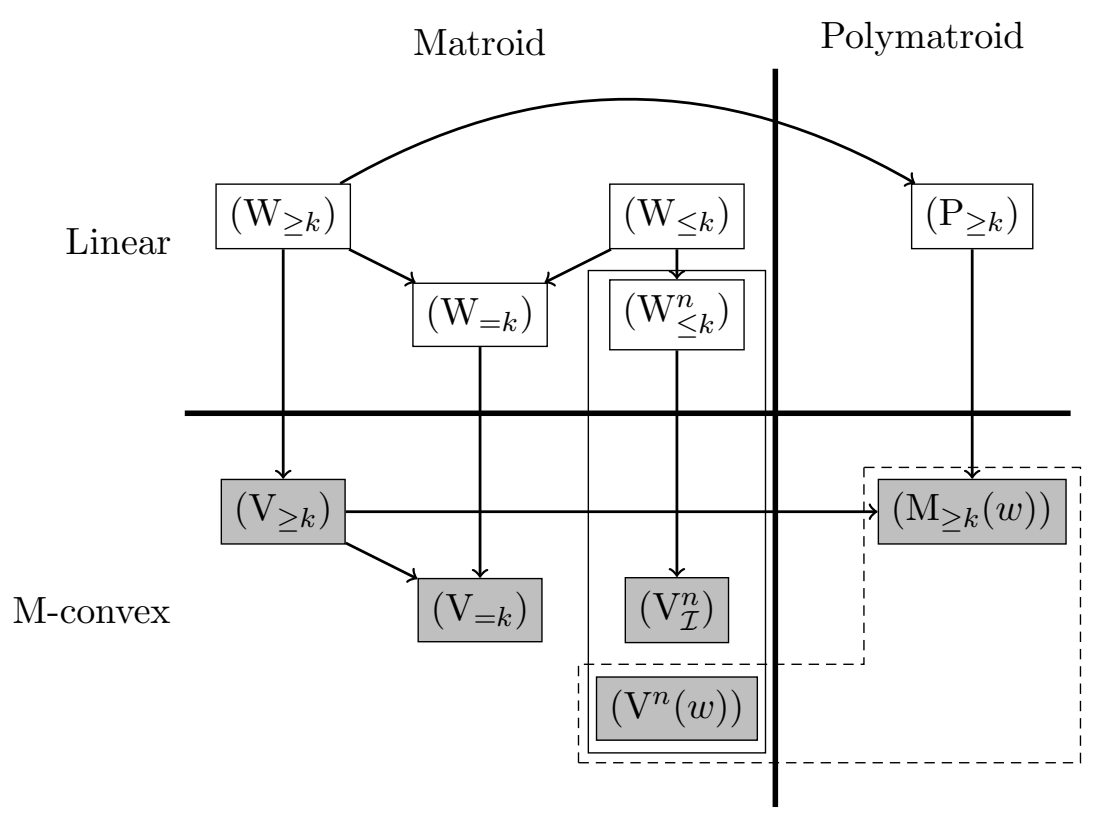

Figure 1: This figure shows the relations among the problems discussed in this paper. The problems filled with gray are new problems introduced in this paper. Each directed solid edge means that the problem at its head is a generalization of that at its tail. The terms "Matroid" and "Polymatroid" in the figure represent that the effective domain of the objective functions of the problem is essentially included in $\{0,1\}^{V}$ and in $\mathbf{Z}^{V}$, respectively. The terms "Linear" and "M-convex" represent that the functions used in the problem are linear (or modular) and M-convex (or valuated matroids), respectively. In $\left(\mathrm{W}_{\leq k}^{n}\right),\left(\mathrm{V}_{\mathcal{I}}^{n}\right)$, and $\left(\mathrm{V}^{n}(w)\right)$, which are included in the solid rectangle, more than two modular functions or valuated matroids can appear in the objective function. In $\left(\mathrm{V}^{n}(w)\right)$ and $\left(\mathrm{M}_{\geq k}(w)\right)$, which are included in the dotted polygon, an additional modular/linear function $w$ appears in the objective function.

- It is also reasonable to take the intersection constraint into the objective function. Let $w: V \rightarrow \mathbf{R}$ be a weight function. The next problem is a variant of $\left(\mathrm{V}_{\mathcal{I}}^{n}\right)$.

$$
\left(\mathrm{V}^{n}(w)\right) \quad \text { Minimize } \quad \sum_{i=1}^{n} \omega_{i}\left(X_{i}\right)+w\left(\bigcap_{i=1}^{n} X_{i}\right) .
$$

- Let $f_{1}$ and $f_{2}$ be M-convex functions on $\mathbf{Z}^{V}$ such that $\operatorname{dom} f_{1}$ and $\operatorname{dom} f_{2}$ form the base polytopes of some polymatroids. Also let $w: \mathbf{Z}^{V} \rightarrow \mathbf{R}$ be a linear function. Then, the following problem is a common generalization of $\left(\mathrm{P}_{\geq k}\right)$ and $\left(\mathrm{V}_{\geq k}\right)$.

$$
\begin{array}{lll}
\left(\mathrm{M}_{\geq k}(w)\right) & \begin{array}{l}
\text { Minimize } \\
\text { subject to }
\end{array} & f_{1}\left(x_{1}\right)+f_{2}\left(x_{2}\right)+w\left(\min \left\{x_{1}, x_{2}\right\}\right) \\
& & \min \left\{x_{1}(v), x_{2}(v)\right\} \geq k,
\end{array}
$$

where $\min \left\{x_{1}, x_{2}\right\} \in \mathbf{Z}^{V}$ is a vector defined by $\min \left\{x_{1}, x_{2}\right\}=\left(\min \left\{x_{1}(v), x_{2}(v)\right\}\right)_{v \in V}$.

The relations among the above problems are given in Figure 1.

Our main contribution is to show the tractability of these generalized problems.

Theorem 1. There exist strongly polynomial-time algorithms to solve $\left(\mathrm{V}_{=k}\right),\left(\mathrm{V}_{\geq k}\right),\left(\mathrm{V}_{\mathcal{I}}^{n}\right)$, and $\left(\mathrm{V}^{n}(w)\right)$ for $w \geq 0$, and a weakly polynomial-time algorithm to solve $\left(\mathrm{M}_{\geq k}(w)\right)$ for $w \leq 0$. 
The algorithms for $\left(\mathrm{V}_{=k}\right)$ and $\left(\mathrm{V}_{\geq k}\right)$ are based on valuated independent assignment [27, 28], that for $\left(\mathrm{V}_{\mathcal{I}}^{n}\right)$ and $\left(\mathrm{V}^{n}(w)\right)$ on valuated matroid intersection $[27,28]$, and that for $\left(\mathrm{M}_{\geq k}(w)\right)$ on $M^{\natural}$-convex submodular flow [30]. We remark that valuated independent assignment generalizes valuated matroid intersection, and $\mathrm{M}^{\natural}$-convex submodular flow is a further generalization. Besides this, the following facts are of theoretical interest.

- If we apply our algorithm for $\left(\mathrm{V}_{=k}\right)$ to the special case $\left(\mathrm{W}_{=k}\right)$, we obtain a primal-dual algorithm which is essentially the same as that in [24], but builds upon a slightly different optimality condition. Details are described in Section 3.2.

- For $\left(\mathrm{V}^{n}(w)\right)$ with $w \geq 0$, our reduction results in a problem which is beyond weighted matroid intersection even if $\omega_{i}$ is a weight function on $V$ with a size constraint, i.e., $\omega_{i}$ is described as

$$
\omega_{i}(X)= \begin{cases}\sum_{v \in X} \omega_{i}(v) & \text { if }|X|=r_{i}, \\ +\infty & \text { if }|X| \neq r_{i}\end{cases}
$$

with some nonnegative integer $r_{i}$ for each $i \in[n]$. That is, this special case of $\left(\mathrm{V}^{n}(w)\right)$ is of interest in the sense that it does not require matroids to define, but requires valuated matroids to solve.

- It might also be interesting that $\left(\mathrm{V}_{\mathcal{I}}^{n}\right)$ can be solved in polynomial time when $n \geq 3$, in spite of the fact that matroid intersection for more than two matroids is NP-hard.

We also demonstrate that the tractability of $\left(\mathrm{V}^{n}(w)\right)$ and $\left(\mathrm{M}_{\geq k}(w)\right)$ relies on the assumptions on $w$ ( $w \geq 0$ and $w \leq 0$, respectively), by showing their NP-hardness for the general case.

Theorem 2. Problems $\left(\mathrm{V}^{n}(w)\right)$ and $\left(\mathrm{M}_{\geq k}(w)\right)$ are NP-hard in general.

We then present applications of our generalized problems to the recoverable robust matroid basis problem, combinatorial optimization problems with interaction costs (COPIC) [23], and matroid congestion games [1]. First we provide a generalization of a certain class of the recoverable robust matroid basis problem in which the cost functions are M-convex functions. This is a special case of $\left(\mathrm{M}_{\geq k}(w)\right)$, and thus can be solved in polynomial time. We next reduce a certain generalized case of the COPIC with diagonal costs to $\left(\mathrm{V}^{n}(w)\right)$ and $\left(\mathrm{M}_{\geq k}(w)\right)$, to provide a generalized class of COPIC which can be solved in polynomial time. Finally, we show that computing the socially optimal state in a certain generalized model of matroid congestion games can be reduced to (a generalized version of) $\left(\mathrm{V}^{n}(w)\right)$ for $w \geq 0$, and thus can be done in polynomial time.

The rest of the paper is organized as follows. Section 2 provides several fundamental facts on valuated matroids and M-convex functions. In Section 3, we present algorithms for solving $\left(\mathrm{V}_{=k}\right)$ and $\left(\mathrm{V}_{\geq k}\right)$ based on a valuated independent assignment algorithm. Sections 4 and 5 are devoted to the reductions of $\left(\mathrm{V}_{\mathcal{I}}^{n}\right)$ and $\left(\mathrm{V}^{n}(w)\right)$ for $w \geq 0$ to valuated matroid intersection, and $\left(\mathrm{M}_{\geq k}(w)\right)$ for $w \leq 0$ to $\mathrm{M}^{\natural}$-convex submodular flow, respectively. We then prove that $\left(\mathrm{V}^{n}(w)\right)$ and $\left(\mathrm{M}_{\geq k}(w)\right)$ are in general NP-hard in Section 6. In Section 7, we present applications of our generalized problems in recoverable robust matroid basis problems, combinatorial optimization problems with interaction costs, and matroid congestion games. Finally, in Section 8, we pose open problems which look similar to those discussed in this paper. 


\section{Preliminaries}

We prepare several facts and terminologies on valuated matroids and M-convex functions. We have already used $\mathbf{Z}$ and $\mathbf{R}$ to denote the sets of integers and real numbers, respectively. The set of nonnegative integers are denoted by $\mathbf{Z}_{+}$, and those of nonnegative real numbers and nonpositive real numbers by $\mathbf{R}_{+}$, and $\mathbf{R}_{-}$, respectively. Recall the definition of M-convex functions described in Section 1. For an M-convex function $f$, all members in $\operatorname{dom} f$ have the same "cardinality," that is, there exists some integer $r$ such that $\sum_{v \in V} x(v)=r$ for all $x \in \operatorname{dom} f$. We refer to $r$ as the rank of $f$. In $\left(\mathrm{M}_{\geq k}(w)\right)$, we require that $\operatorname{dom} f_{1}$ and $\operatorname{dom} f_{2}$ form the base polytopes of some polymatroids. This condition is equivalent to $\operatorname{dom} f_{1} \subseteq \mathbf{Z}_{+}^{V}$ and $\operatorname{dom} f_{2} \subseteq \mathbf{Z}_{+}^{V}$.

Recall that a valuated matroid is an M-convex function defined on $2^{V}$. Valuated matroid intersection $[27,28]$ is a generalization of weighted matroid intersection defined as follows: Given two valuated matroids $\omega_{1}$ and $\omega_{2}$ on $2^{V}$, find $X \subseteq V$ minimizing the sum $\omega_{1}(X)+\omega_{2}(X)$.

We next define the valuated independent assignment problem $[27,28]$. Let $G=\left(V_{1}, V_{2} ; E\right)$ be a bipartite graph, $\omega_{1}: 2^{V_{1}} \rightarrow \mathbf{R} \cup\{+\infty\}$ and $\omega_{2}: 2^{V_{2}} \rightarrow \mathbf{R} \cup\{+\infty\}$ be valuated matroids, and $w: E \rightarrow \mathbf{R}$ be a weight function. The valuated independent assignment problem parameterized by an integer $k$, referred to as $\operatorname{VIAP}(k)$, is described as follows.

$$
\begin{array}{lll} 
& \text { Minimize } & \omega_{1}\left(X_{1}\right)+\omega_{2}\left(X_{2}\right)+w(F) \\
\operatorname{VIAP}(k) \quad & \text { subject to } & F \subseteq E \text { is a matching of } G \text { with } \partial F \subseteq X_{1} \cup X_{2}, \\
& |F|=k,
\end{array}
$$

where $\partial F$ denote the set of the endpoints of $F \subseteq E$. As mentioned in Section $1, \operatorname{VIAP}(k)$ is a generalization of valuated matroid intersection, and both of them can be solved in strongly polynomial time [27, 28].

A function $f: \mathbf{Z}^{V} \rightarrow \mathbf{R} \cup\{+\infty\}$ is said to be $M^{\natural}$-convex [35] if it satisfies the following weaker exchange axiom: for all $x=(x(v))_{v \in V}$ and $y=(y(v))_{v \in V}$ with $x, y \in \operatorname{dom} f$, and all $v \in V$ with $x(v)>y(v)$, it holds that

$$
f(x)+f(y) \geq f\left(x-\chi_{v}\right)+f\left(y+\chi_{v}\right),
$$

or there exists $u \in V$ with $x(v)<y(v)$ such that

$$
f(x)+f(y) \geq f\left(x-\chi_{v}+\chi_{u}\right)+f\left(y+\chi_{v}-\chi_{u}\right) .
$$

It is clear from the definition that $\mathrm{M}^{\natural}$-convexity slightly generalizes $\mathrm{M}$-convexity, while they are known to be essentially equivalent (see, e.g., [32], for details). The following lemma shows one relation between $\mathrm{M}$-convex and $\mathrm{M}^{\natural}$-convex functions.

Lemma 3 ([35]). For an $M^{\natural}$-convex function $f$ and an integer $r$, the restriction of $f$ to a hyperplane $\left\{x \in \mathbf{Z}^{V} \mid \sum_{v \in V} x(v)=r\right\}$ is an $M$-convex function with rank $r$, if its effective domain is nonempty.

We close this section with the definition of $M^{\natural}$-convex submodular flow [30]. Let $f$ be an $\mathrm{M}^{\natural}$-convex function on $\mathbf{Z}^{V}$ and $G=(V, A)$ a directed graph endowed with an upper capacity function $\bar{c}: A \rightarrow \mathbf{R} \cup\{+\infty\}$, a lower capacity function $\underline{c}: A \rightarrow \mathbf{R} \cup\{-\infty\}$, and a weight function $w: A \rightarrow \mathbf{R}$. For a vector $\xi \in \mathbf{R}^{A}$, define its boundary $\partial \xi \in \mathbf{R}^{V}$ by

$$
\partial \xi(v):=\sum\{\xi(a) \mid a \in A, a \text { enters } v \text { in } G\}-\sum\{\xi(a) \mid a \in A, a \text { leaves } v \text { in } G\}
$$

for $v \in V$. The $M^{\natural}$-convex submodular flow problem for $(f, G)$ is the following problem with variable $\xi \in \mathbf{R}^{A}$ :

$$
\begin{array}{ll}
\text { Minimize } & f(\partial \xi)+\sum_{a \in A} w(a) \xi(a) \\
\text { subject to } & \underline{c}(a) \leq \xi(a) \leq \bar{c}(a) .
\end{array}
$$


The $\mathrm{M}^{\natural}$-convex submodular flow problem is a further generalization of $\operatorname{VIAP}(k)$, and can be solved in weakly polynomial time [17, 18].

\section{Solving $\left(\mathrm{V}_{=k}\right)$ and $\left(\mathrm{V}_{\geq k}\right)$ via valuated independent assignment}

This section provides strongly polynomial-time algorithms for solving $\left(\mathrm{V}_{=k}\right)$ and $\left(\mathrm{V}_{\geq k}\right)$. For their special cases $\left(\mathrm{W}_{=k}\right)$ and $\left(\mathrm{W}_{\geq k}\right)$, Lendl et al. [24] showed the polynomial-time solvability: they developed a new algorithm specific to $\left(\mathrm{W}_{=k}\right)$, and reduced $\left(\mathrm{W}_{\geq k}\right)$ to weighted matroid intersection. In this paper, building upon the DCA perspective, we show that both of the generalized problems $\left(\mathrm{V}_{=k}\right)$ and $\left(\mathrm{V}_{\geq k}\right)$ fall in the framework of valuated independent assignment.

\subsection{Strongly polynomial-time algorithms}

We first present an algorithm for $\left(\mathrm{V}_{\geq k}\right)$. Given an instance of $\left(\mathrm{V}_{\geq k}\right)$, construct an instance of $\operatorname{VIAP}(k)$ as follows. Set a bipartite graph $G$ by $\left(V_{1}, V_{2} ;\left\{\left\{v^{1}, v^{2}\right\} \mid v \in V\right\}\right)$, where $V_{i}$ is a copy of $V$ and $v^{i} \in V_{i}$ is a copy of $v \in V$ for $i=1,2$. By abuse of notation, for $i=1,2$, a subset $X_{i}$ of $V$ is regarded as a subset of $V_{i}$ as well, and $\omega_{i}$ is regarded as a valuated matroid on $2^{V_{i}}$. Set $w(e):=0$ for every edge $e$. We now obtain an instance of $\operatorname{VIAP}(k)$ defined by $G, \omega_{1}, \omega_{2}$, and $w$.

One can see that, if $\left(X_{1}, X_{2}\right)$ is feasible for $\left(\mathrm{V}_{\geq k}\right)$, i.e., $\left|X_{1} \cap X_{2}\right| \geq k$, then there is a matching $F$ of $G$ with $\partial F \subseteq X_{1} \cup X_{2}$ and $|F|=k$, i.e., there exists a feasible solution $\left(X_{1}, X_{2}, F\right)$ for $\operatorname{VIAP}(k)$. On the other hand, if $\left(X_{1}, X_{2}, F\right)$ is a feasible solution for $\operatorname{VIAP}(k)$, then $\left(X_{1}, X_{2}\right)$ is feasible for $\left(\mathrm{V}_{\geq k}\right)$. Moreover the objective value of a feasible solution $\left(X_{1}, X_{2}\right)$ for $\left(\mathrm{V}_{\geq k}\right)$ is equal to that of any corresponding feasible solution $\left(X_{1}, X_{2}, F\right)$ for $\operatorname{VIAP}(k)$ since $w(e)$ is identically zero.

Thus, $\left(\mathrm{V}_{\geq k}\right)$ reduces to $\operatorname{VIAP}(k)$, and hence can be solved in strongly polynomial time in the following way based on the augmenting path algorithm for $\operatorname{VIAP}(k)$ [27, 28]; see also [31, Theorem 5.2.62]. Here let $X_{1}$ and $X_{2}$ be the minimizers of $\omega_{1}$ and $\omega_{2}$, respectively, which can be found in a greedy manner.

Step 1: If $\left|X_{1} \cap X_{2}\right| \geq k$, then output $\left(X_{1}, X_{2}\right)$ and stop. Otherwise, let $X_{1}^{j}:=X_{1}$ and $X_{2}^{j}:=X_{2}$, where $j:=\left|X_{1} \cap X_{2}\right|<k$.

Step 2: Execute the augmenting path algorithm for $\operatorname{VIAP}(k)$. Then we obtain a sequence $\left(\left(X_{1}^{j}, X_{2}^{j}\right),\left(X_{1}^{j+1}, X_{2}^{j+1}\right), \ldots,\left(X_{1}^{\ell}, X_{2}^{\ell}\right)\right)$ of solutions, where $\left|X_{1}^{j^{\prime}} \cap X_{2}^{j^{\prime}}\right|=j^{\prime}$ for $j^{\prime}=j, j+$ $1, \ldots, \ell$. If $\ell<k$, then output " $\left(\mathrm{V}_{\geq k}\right)$ is infeasible." If $\ell \geq k$, then output $\left(X_{1}^{k}, X_{2}^{k}\right)$.

We describe the full behavior of the above algorithm in Appendix A.

Our algorithm for $\left(\mathrm{V}_{=k}\right)$ directly follows from this algorithm for $\left(\mathrm{V}_{\geq k}\right)$. Again let $X_{1}$ and $X_{2}$ be the minimizers of $\omega_{1}$ and $\omega_{2}$, respectively.

Case $1\left(\left|X_{1} \cap X_{2}\right| \leq k\right)$ : Execute the augmenting path algorithm for $\left(\mathrm{V}_{\geq k}\right)$. If the algorithm detects the infeasibility of $\left(\mathrm{V}_{\geq k}\right)$, then output " $\left(\mathrm{V}_{=k}\right)$ is infeasible." Otherwise we obtain an optimal solution $\left(X_{1}^{*}, X_{2}^{*}\right)$ with $\left|X_{1}^{*} \cap X_{2}^{*}\right|=k$ for $\left(\mathrm{V}_{\geq k}\right)$, and output it.

Case $2\left(\left|X_{1} \cap X_{2}\right|>k\right)$ : Let $r_{1}$ be the rank of $\omega_{1}$ and $\overline{\omega_{2}}(X):=\omega_{2}(V \backslash X)$ for $X \subseteq V$, which is the dual valuated matroid of $\omega_{2}$. Note that $X_{1}$ and $V \backslash X_{2}$ are minimizers of $\omega_{1}$ and $\overline{\omega_{2}}$, respectively, and $X_{1} \cap\left(V \backslash X_{2}\right)<r_{1}-k$. Then apply Case 1 to $\left(\mathrm{V}_{=r_{1}-k}\right)$ for $\omega_{1}$ and $\overline{\omega_{2}}$.

It is clear that the above methods solve $\left(\mathrm{V}_{\geq k}\right)$ and $\left(\mathrm{V}_{=k}\right)$ in strongly polynomial time. We can provide the explicit time complexities as follows. The proof is deferred to Appendix A. 
Theorem 4. Problems $\left(\mathrm{V}_{\geq k}\right)$ and $\left(\mathrm{V}_{=k}\right)$ can be solved in $O(|V| r k \gamma+|V| k \log |V|)$ time and $O\left(|V| r^{2} \gamma+|V| r \log |V|\right)$ time, respectively, where $r$ is the maximum of the ranks of $\omega_{1}$ and $\omega_{2}$ and $\gamma$ is the time required for computing the function value.

Remark 5. If we are given at least three valuated matroids, then $\left(\mathrm{V}_{=k}\right)$ and $\left(\mathrm{V}_{\geq k}\right)$ (even $\left(\mathrm{W}_{=k}\right)$ and $\left.\left(\mathrm{W}_{\geq k}\right)\right)$ will be NP-hard, since they can formulate the matroid intersection problem for three matroids. Indeed, let $M_{1}=\left(V, \mathcal{B}_{1}\right), M_{2}=\left(V, \mathcal{B}_{2}\right), M_{3}=\left(V, \mathcal{B}_{3}\right)$ be matroids with the same rank $r$. Then it is clear that there exist $X_{1}, X_{2}, X_{3} \subseteq V$ with $X_{1} \in \mathcal{B}_{1}, X_{2} \in \mathcal{B}_{2}, X_{3} \in \mathcal{B}_{3}$ and $\left|X_{1} \cap X_{2} \cap X_{3}\right| \geq r$ (or $\left|X_{1} \cap X_{2} \cap X_{3}\right|=r$ ) if and only if there exists $X \subseteq V$ with $X \in \mathcal{B}_{1} \cap \mathcal{B}_{2} \cap \mathcal{B}_{3}$.

We close this subsection by exhibiting two more solutions to $\left(\mathrm{V}_{\geq k}\right)$ and $\left(\mathrm{V}_{=k}\right)$. The first one is a different reduction of $\left(\mathrm{V}_{\geq k}\right)$ to valuated matroid intersection, which requires the argument described in Section 4 below. Indeed, $\left(\mathrm{V}_{\geq k}\right)$ for $\omega_{1}$ and $\omega_{2}$ is equivalent to $\left(\mathrm{V}_{\leq r_{1}-k}\right)$ for $\omega_{1}$ and $\overline{\omega_{2}}$, where $r_{1}$ is the rank of $\omega_{1}$ and $\overline{\omega_{2}}$ is the dual valuated matroid of $\omega_{2}$. One can see that $\left(\mathrm{V}_{\leq r_{1}-k}\right)$ is a special case of $\left(\mathrm{V}_{\mathcal{I}}^{n}\right)$ in which $n=2$ and $\mathcal{I}$ is the independent set family of the uniform matroid with rank $r_{1}-k$. Thus, by the argument in Section $4,\left(\mathrm{~V}_{\leq r_{1}-k}\right)$ can be viewed as a special instance of valuated matroid intersection.

The second one is a solution to $\left(\mathrm{V}_{=k}\right)$. Recently, László Végh [40] has presented a simple $O\left(|V| r^{2} \gamma+|V| r \log |V|\right)$-time algorithm for $\left(\mathrm{W}_{=k}\right)$. By extending this algorithm, we can obtain another solution to $\left(\mathrm{V}_{=k}\right)$, which is described in Appendix B.

\subsection{Relation to the algorithm by Lendl et al.}

In this subsection, we illustrate the similarity and difference between the algorithm of Lendl et al. [24] for $\left(\mathrm{W}_{=k}\right)$ and our algorithm applied to $\left(\mathrm{W}_{=k}\right)$. For a detailed description of the algorithm of Lendl et al. [24], the readers are referred to Appendix C.

As is shown in Appendices $\mathrm{A}$ and $\mathrm{C}$, both algorithms maintain potential functions on $V_{1}$ and $V_{2}$. Main differences appear in the optimality criteria, and in the updating procedures of a solution and potentials.

The algorithm of Lendl et al. [24] is based on the following sufficient condition of the optimality: A feasible solution $\left(X_{1}, X_{2}\right)$ of $\left(\mathrm{W}_{=k}\right)$ is optimal if there exist a nonnegative potential function $q_{1}: V_{1} \rightarrow \mathbf{R}_{+}$, a nonpositive potential function $q_{2}: V_{2} \rightarrow \mathbf{R}_{-}$, and a nonnegative value $\lambda \in \mathbf{R}_{+}$satisfying that

- $q_{1}\left(v^{1}\right)=q_{2}\left(v^{2}\right)+\lambda$ for each $v \in V$,

- $X_{1}$ and $X_{2}$ are minimizers of $w_{1}-q_{1}$ and $w_{2}+q_{2}$, respectively, and

- $q_{1}\left(v^{1}\right)=0$ for $v^{1} \in X_{1} \backslash X_{2}$ and $q_{2}\left(v^{2}\right)=0$ for $v^{2} \in X_{2} \backslash X_{1}$.

We refer to such $\left(q_{1}, q_{2}\right)$ as an LPT optimality witness of $\left(X_{1}, X_{2}\right)$.

Our algorithm is based on the following is the optimality criteria for $\operatorname{VIAP}(k)$ in $[27$, Theorem 5.1] specialized to $\left(\mathrm{V}_{\geq k}\right)$. For a set function $\omega$ on $2^{V}$ and a weight function $w$ on $V$, we define the functions $\omega+p$ and $\omega-p$ on $2^{V}$ by

$$
\begin{aligned}
& (\omega+p)(X):=\omega(X)+p(X) \\
& (\omega-p)(X):=\omega(X)-p(X)
\end{aligned}
$$

for each $X \subseteq V$.

Lemma 6. A feasible solution $\left(X_{1}, X_{2}\right)$ for $\left(\mathrm{V}_{\geq k}\right)$ is optimal if and only if there are potential functions $p_{1}: V_{1} \rightarrow \mathbf{R}$ and $p_{2}: V_{2} \rightarrow \mathbf{R}$ satisfying the following three conditions: 
- $p_{1}\left(v^{1}\right)=p_{2}\left(v^{2}\right)$ for each $v \in V$;

- $X_{1}$ and $X_{2}$ are minimizers of $\omega_{1}-p_{1}$ and $\omega_{2}+p_{2}$, respectively; and

- there is $F \subseteq X_{1} \cap X_{2}$ such that $|F|=k, X_{1} \backslash F \subseteq \operatorname{argmin} p_{1}$, and $X_{2} \backslash F \subseteq \operatorname{argmax} p_{2}$.

For an optimal solution $\left(X_{1}, X_{2}\right)$ for $\left(\mathrm{V}_{\geq k}\right)$, we refer to a triple $\left(p_{1}, p_{2}, F\right)$ satisfying the three conditions in Lemma 6 as an optimality witness of $\left(X_{1}, X_{2}\right)$.

We first see that the potential functions in the algorithm of Lendl et al. and those in our algorithm are essentially equivalent. During our algorithm for $\left(\mathrm{V}_{\geq k}\right)$, one can see that the potential functions $p_{1}$ and $p_{2}$ particularly satisfy the following:

- $p_{1}\left(v^{1}\right)=p_{2}\left(v^{2}\right)$ for each $v \in V$.

- $X_{1}$ and $X_{2}$ are minimizers of $\omega_{1}-p_{1}$ and $\omega_{2}+p_{2}$, respectively.

- $\min p_{1}=0, X_{1} \backslash X_{2} \subseteq \operatorname{argmin} p_{1}$, and $X_{2} \backslash X_{1} \subseteq \operatorname{argmax} p_{2}$.

Hence, if $\left(q_{1}, q_{2}\right)$ is an LPT optimality witness, then $\left(p_{1}, p_{2}\right)$ with $p_{1}=p_{2}=q_{1}$ forms an optimality witness in our sense. Conversely, if $\left(p_{1}, p_{2}\right)$ is an optimality witness appearing in our algorithm, then $\left(q_{1}, q_{2}\right)$ with $q_{1}=p_{1}$ and $q_{2}=p_{2}-\lambda$ forms an LPT optimality witness for $\lambda:=\max p_{2}$.

We next sketch the difference in the updating procedures, whose details are discussed in Appendix C. The update phases of a solution and of potential functions in the algorithm of Lendl et al. are completely separated, while our algorithm simultaneously updates a solution and potential functions. This difference in the updating procedures leads to the difference in the running-times of the algorithms; the algorithm of Lendl et al. runs in $O\left(|V|^{2} r^{2}\right)$ time, and our algorithm in $O\left(|V| r^{2}+|V| r \log |V|\right)$ time by Theorem 4 with $\gamma=O(1)$, which is better than that of Lendl et al.

\section{Reducing $\left(\mathrm{V}_{\mathcal{I}}^{n}\right)$ and $\left(\mathrm{V}^{n}(w)\right)$ to valuated matroid intersection}

In this section, we present reductions of $\left(\mathrm{V}_{\mathcal{I}}^{n}\right)$ and $\left(\mathrm{V}^{n}(w)\right)$ for $w \geq 0$ to valuated matroid intersection, which implies strongly polynomial-time algorithms for those problems. We begin with the following result for valuated matroid intersection.

Lemma 7 ([28]; see also Theorem 4). Let $\omega$ and $\omega^{\prime}$ be valuated matroids on $2^{V}$ with rank $r$ and $\gamma$ the time required for computing the function value. Valuated matroid intersection for $\omega$ and $\omega^{\prime}$ can be solved in $O\left(|V| r^{2} \gamma+|V| r \log |V|\right)$ time.

For the reductions, we need to prepare a pair of valuated matroids for each problem. One valuated matroid is common in the reductions of $\left(\mathrm{V}_{\mathcal{I}}^{n}\right)$ and $\left(\mathrm{V}^{n}(w)\right)$, which is defined as follows. Let $V_{1}, V_{2}, \ldots, V_{n}$ be $n$ disjoint copies of $V$, and let $\tilde{V}=\bigcup_{i \in[n]} V_{i}$. By abuse of notation, for $n$ subsets $X_{1}, X_{2}, \ldots, X_{n} \subseteq V$, we denote by $\left(X_{1}, X_{2}, \ldots, X_{n}\right)$ a subset of $\tilde{V}$ composed of the copies of $X_{i}$ included in $V_{i}(i \in[n])$. Let us define a valuated matroid $\tilde{\omega}$ by the disjoint sum of $\omega_{1}, \omega_{2}, \ldots, \omega_{n}$. That is, $\tilde{\omega}$ is a function on $2^{\tilde{V}}$ defined by

$$
\tilde{\omega}\left(X_{1}, X_{2}, \ldots, X_{n}\right):=\omega_{1}\left(X_{1}\right)+\omega_{2}\left(X_{2}\right)+\cdots+\omega_{n}\left(X_{n}\right)
$$

for each $\left(X_{1}, X_{2}, \ldots, X_{n}\right) \subseteq \tilde{V}$. It follows that $\tilde{\omega}$ is a valuated matroid with rank $r:=\sum_{i=1}^{n} r_{i}$, where $r_{i}$ is the rank of $\omega_{i}$. 
We then provide the other valuated matroid used in the reduction of $\left(\mathrm{V}_{\mathcal{I}}^{n}\right)$. Define a set system $\tilde{M}=(\tilde{V}, \tilde{\mathcal{B}})$ by

$$
\tilde{\mathcal{B}}=\left\{\left(X_{1}, X_{2}, \ldots, X_{n}\right)\left|X_{i} \subseteq V(i \in[n]), \bigcap_{i=1}^{n} X_{i} \in \mathcal{I}, \sum_{i=1}^{n}\right| X_{i} \mid=r\right\} .
$$

It is clear that $\left(\mathrm{V}_{\mathcal{I}}^{n}\right)$ amounts to minimizing the sum of $\tilde{\omega}$ and $\delta_{\tilde{\mathcal{B}}}$, where $\delta_{\tilde{\mathcal{B}}}$ denotes the indicator function of $\tilde{\mathcal{B}}$, namely,

$$
\delta_{\tilde{\mathcal{B}}}\left(X_{1}, X_{2}, \ldots, X_{n}\right)= \begin{cases}0 & \text { if }\left(X_{1}, X_{2}, \ldots, X_{n}\right) \in \tilde{\mathcal{B}} \\ +\infty & \text { otherwise. }\end{cases}
$$

Thus, what remains to be proved is that $\delta_{\tilde{\mathcal{B}}}$ is a valuated matroid, and is derived from the following lemma.

Lemma 8. The set system $\tilde{M}=(\tilde{V}, \tilde{\mathcal{B}})$ is a matroid with base family $\tilde{\mathcal{B}}$.

Proof. Let $\tilde{\mathcal{I}} \subseteq \tilde{V}$ be a subset family obtained from $\tilde{\mathcal{B}}$ by removing the cardinality condition, that is,

$$
\tilde{\mathcal{I}}=\left\{\left(X_{1}, X_{2}, \ldots, X_{n}\right) \mid X_{i} \subseteq V(i \in[n]), \bigcap_{i=1}^{n} X_{i} \in \mathcal{I}\right\}
$$

It suffices to show that $\tilde{\mathcal{I}}$ is an independent set family of a matroid, i.e., $\tilde{\mathcal{I}}$ satisfies the following axioms:

- $(\emptyset, \emptyset, \ldots, \emptyset) \in \tilde{\mathcal{I}}$.

- $\left(X_{1}, X_{2}, \ldots, X_{n}\right) \subseteq\left(Y_{1}, Y_{2}, \ldots, Y_{n}\right) \in \tilde{\mathcal{I}}$ implies $\left(X_{1}, X_{2}, \ldots, X_{n}\right) \in \tilde{\mathcal{I}}$.

- For every $\left(X_{1}, X_{2}, \ldots, X_{n}\right),\left(Y_{1}, Y_{2}, \ldots, Y_{n}\right) \in \tilde{\mathcal{I}}$ with $\sum_{i=1}^{n}\left|X_{i}\right|<\sum_{i=1}^{n}\left|Y_{i}\right|$, there exist $i^{*} \in[n]$ and $v^{*} \in Y_{i^{*}} \backslash X_{i^{*}}$ such that $\left(X_{1}, \ldots, X_{i^{*}} \cup\left\{v^{*}\right\}, \ldots, X_{n}\right) \in \tilde{\mathcal{I}}$.

The first and second are clear. We prove the third. If there exist $i^{*} \in[n]$ and $v^{*} \in Y_{i^{*}} \backslash X_{i^{*}}$ such that

$$
\left(\bigcap_{i \in[n] \backslash\left\{i^{*}\right\}} X_{i}\right) \cap\left(X_{i^{*}} \cup\left\{v^{*}\right\}\right)=\bigcap_{i=1}^{n} X_{i}
$$

then $\left(X_{1}, \ldots, X_{i^{*}} \cup\left\{v^{*}\right\}, \ldots, X_{n}\right) \in \tilde{\mathcal{I}}$ follows from $\bigcap_{i=1}^{n} X_{i} \in \mathcal{I}$.

Suppose that such $i^{*}$ and $v^{*}$ do not exist. For each $v \in V$, denote by $X^{(v)}$ (resp. $Y^{(v)}$ ) the set of indices $i \in[n]$ with $v \in X_{i}$ (resp. $v \in Y_{i}$ ). Then, for each $v \in V$, we have $X^{(v)} \supseteq Y^{(v)}$ or $X^{(v)}=[n] \backslash\{i\}$ for some $i \in[n]$. This implies that $\left|Y^{(v)}\right|>\left|X^{(v)}\right|$ if and only if $Y^{(v)}=[n]$ and $X^{(v)}=[n] \backslash\{i\}$ for some $i$. It then follows from $\sum_{i=1}^{n}\left|X_{i}\right|=\sum_{v \in V}\left|X^{(v)}\right|, \sum_{i=1}^{n}\left|Y_{i}\right|=$ $\sum_{v \in V}\left|Y^{(v)}\right|$, and $\sum_{i=1}^{n}\left|X_{i}\right|<\sum_{i=1}^{n}\left|Y_{i}\right|$ that

$$
\begin{aligned}
\left|\left(\bigcap_{i=1}^{n} Y_{i}\right) \backslash\left(\bigcap_{i=1}^{n} X_{i}\right)\right| & =\left|\left\{v \in V \mid Y^{(v)}=[n], X^{(v)} \subsetneq[n]\right\}\right| \\
& =\sum_{v \in V,\left|Y^{(v)}\right|>\left|X^{(v)}\right|}\left(\left|Y^{(v)}\right|-\left|X^{(v)}\right|\right)
\end{aligned}
$$




$$
\begin{aligned}
& =\sum_{v \in V}\left(\left|Y^{(v)}\right|-\left|X^{(v)}\right|\right)+\sum_{v \in V,\left|X^{(v)}\right|>\left|Y^{(v)}\right|}\left(\left|X^{(v)}\right|-\left|Y^{(v)}\right|\right) \\
& \geq \sum_{i=1}^{n}\left(\left|Y_{i}\right|-\left|X_{i}\right|\right)+\left|\left(\bigcap_{i=1}^{n} X_{i}\right) \backslash\left(\bigcap_{i=1}^{n} Y_{i}\right)\right| \\
& >\left|\left(\bigcap_{i=1}^{n} X_{i}\right) \backslash\left(\bigcap_{i=1}^{n} Y_{i}\right)\right| \\
& \geq 0 .
\end{aligned}
$$

Since $\bigcap_{i=1}^{n} X_{i}$ and $\bigcap_{i=1}^{n} Y_{i}$ belong to $\mathcal{I}$, there exists $v^{*} \in\left(\bigcap_{i=1}^{n} Y_{i}\right) \backslash\left(\bigcap_{i=1}^{n} X_{i}\right)$ such that $\left(\bigcap_{i=1}^{n} X_{i}\right) \cup\left\{v^{*}\right\} \in \mathcal{I}$. Let $i^{*} \in[n]$ be an index such that $v^{*} \in Y_{i^{*}} \backslash X_{i^{*}}$. We then obtain $\left(X_{1}, \ldots, X_{i^{*}} \cup\left\{v^{*}\right\}, \ldots, X_{n}\right) \in \mathcal{I}$, since $\left(\bigcap_{i \in[n] \backslash\left\{i^{*}\right\}} X_{i}\right) \cap\left(X_{i^{*}} \cup\left\{v^{*}\right\}\right)=\left(\bigcap_{i=1}^{n} X_{i}\right) \cup\left\{v^{*}\right\} \in \mathcal{I}$.

It follows from Lemma 8 that the function $\delta_{\tilde{\mathcal{B}}}$ is a valuated matroid, and we conclude that $\left(\mathrm{V}_{\mathcal{I}}^{n}\right)$ can be reduced to valuated matroid intersection. Thus we obtain the following theorem from Lemma 7:

Theorem 9. Problem $\left(\mathrm{V}_{\mathcal{I}}^{n}\right)$ can be solved in $O\left(|V| n r^{2} \gamma+|V| n r \log (|V| n)\right)$ time, where $\gamma$ is the time required for computing the function value.

Remark 10. If we replace the constraint $\bigcap_{i=1}^{n} X_{i} \in \mathcal{I}$ in $\left(\mathrm{V}_{\mathcal{I}}^{n}\right)$ by $\bigcap_{i=1}^{n} X_{i} \in \mathcal{B}$, where $\mathcal{B}$ is the base family of some matroid, then the problem will be NP-hard even if $n=2$, since it can formulate the matroid intersection problem for three matroids. In other words, if we replace the intersection constraint $\left|X_{1} \cap X_{2}\right|=k$ in $\left(\mathrm{W}_{=k}\right)$ with $X_{1} \cap X_{2} \in \mathcal{B}$, then the problem becomes NP-hard. Also, recall Remark 5, which implies that the problem is NP-hard if $n \geq 3$ and the constraint is $\left|\bigcap_{i=1}^{n} X_{i}\right|=k$.

We next provide another valuated matroid used in the reduction of $\left(\mathrm{V}^{n}(w)\right)$. A set family $\mathcal{L} \subseteq 2^{V}$ is said to be laminar if $X \subseteq Y, X \supseteq Y$, or $X \cap Y=\emptyset$ holds for all $X, Y \in \mathcal{L}$. A function $f: \mathbf{Z}^{V} \rightarrow \mathbf{R} \cup\{+\infty\}$ is said to be laminar convex [32, Section 6.3] if $f$ is representable as

$$
f(x)=\sum_{X \in \mathcal{L}} g_{X}\left(\sum_{v \in X} x(v)\right) \quad\left(x \in \mathbf{Z}^{V}\right),
$$

where $\mathcal{L} \subseteq 2^{V}$ is a laminar family on $V$, and for each $X \in \mathcal{L}, g_{X}: \mathbf{Z} \rightarrow \mathbf{R} \cup\{+\infty\}$ is a univariate discrete convex function, i.e., $g_{X}(k+1)+g_{X}(k-1) \geq 2 g_{X}(k)$ for every $k \in \mathbf{Z}$. A laminar convex function is a typical example of an $\mathrm{M}^{\natural}$-convex function and plays a key role here.

Define a function $\tilde{w}$ on $2^{\tilde{V}}$ by

$$
\tilde{w}\left(X_{1}, X_{2}, \ldots, X_{n}\right):=w\left(\bigcap_{i=1}^{n} X_{i}\right)
$$

for each $\left(X_{1}, X_{2}, \ldots, X_{n}\right) \subseteq \tilde{V}$. It is clear that $\left(\mathrm{V}^{n}(w)\right)$ is equivalent to minimizing the sum of $\tilde{\omega}$ and the restriction of $\tilde{w}$ to $\left\{\left(X_{1}, X_{2}, \ldots, X_{n}\right)\left|\sum_{i=1}^{n}\right| X_{i} \mid=r\right\}$. For the function $\tilde{w}$, the following holds.

Lemma 11. The function $\tilde{w}$ on $2^{\tilde{V}}$ is laminar convex if $w \geq 0$. 
Proof. For each $v \in V$, define a unary function $g_{v}: \mathbf{Z} \rightarrow \mathbf{R}$ by

$$
g_{v}(x):= \begin{cases}w(v) & \text { if } x=n \\ 0 & \text { if } 0 \leq x<n \\ +\infty & \text { otherwise }\end{cases}
$$

It follows from $w(v) \geq 0$ that $g_{v}$ is discrete convex. Moreover, one can see that

$$
\tilde{w}\left(X_{1}, X_{2}, \ldots, X_{n}\right)=\sum_{v \in V} g_{v}\left(\left|\left\{i \in[n] \mid v \in X_{i}\right\}\right|\right) \quad\left(X_{i} \subseteq V, i \in[n]\right)
$$

holds. Here we can regard the right-hand side of (2) as the sum taken for $(\{v\},\{v\}, \ldots,\{v\}) \subseteq \tilde{V}$ for every $v \in V$. Since the family $\{(\{v\},\{v\}, \ldots,\{v\}) \mid v \in V\}$ is laminar on $\tilde{V}$, we conclude that the right-hand side of (2) is a laminar convex function, as required.

By Lemmas 3 and 11 , the restriction of $\tilde{w}$ to $\left\{\left(X_{1}, X_{2}, \ldots, X_{n}\right)\left|\sum_{i=1}^{n}\right| X_{i} \mid=r\right\}$ is a valuated matroid on $2^{\tilde{V}}$ if $w \geq 0$. Thus $\left(\mathrm{V}^{n}(w)\right)$ can be formulated as valuated matroid intersection problem for $\tilde{\omega}$ and $\tilde{w}$, establishing the tractability of $\left(\mathrm{V}^{n}(w)\right)$ in case of $w \geq 0$. That is, we obtain the following theorem from Lemma 7.

Theorem 12. Problem $\left(\mathrm{V}^{n}(w)\right)$ with $w \geq 0$ can be solved in $O\left(|V| n r^{2} \gamma+|V| n r \log (|V| n)\right)$ time, where $\gamma$ is the time required for computing the function value.

Remark 13. As mentioned in Section 1 , even if $\omega_{i}$ is described as (1) for each $i \in[n]$, our reduction goes beyond the weighted matroid intersection framework. This is because the valuated matroid $\tilde{w}$ is not modular regardless whether $\omega_{i}$ is of the form (1). That is, the concept of Mconvexity is essential for us to establish the tractability of $\left(\mathrm{V}^{n}(w)\right)$, even when $\omega_{i}$ is of the form (1).

\section{$5 \quad$ Reducing $\left(\mathrm{M}_{\geq k}(w)\right)$ to $\mathbf{M}^{\natural}$-convex submodular flow}

In this section, we prove that $\left(\mathrm{M}_{\geq k}(w)\right)$ with $w \leq 0$ can be solved in polynomial time by reducing it to $\mathrm{M}^{\mathrm{h}}$-convex submodular flow.

Given an instance of $\left(\mathrm{M}_{\geq k}(w)\right)$ with $w \leq 0$, construct an instance of the $\mathrm{M}^{\natural}$-convex submodular flow problem as follows. Let $V_{1}:=\left\{v^{1} \mid v \in V\right\}$ and $V_{2}:=\left\{v^{2} \mid v \in V\right\}$ be disjoint two copys of $V$. We regard $f_{1}$ and $f_{2}$ as functions on $\mathbf{Z}^{V_{1}}$ and on $\mathbf{Z}^{V_{2}}$, respectively. Recall that $\operatorname{dom} f_{1}$ and $\operatorname{dom} f_{2}$ form the base polytopes of some polymatroids, i.e., $\operatorname{dom} f_{1} \subseteq \mathbf{Z}_{+}^{V}$ and $\operatorname{dom} f_{2} \subseteq \mathbf{Z}_{+}^{V}$. Let $r_{i}$ be the rank of $f_{i}$ for $i=1,2$. We define univariate functions $g_{1}$ and $g_{2}$ on $\mathbf{Z}$ by

$$
g_{1}(p):=\left\{\begin{array}{ll}
0 & \text { if } 0 \leq p \leq r_{2}-k, \\
+\infty & \text { otherwise }
\end{array} \quad g_{2}(q):= \begin{cases}0 & \text { if } 0 \leq q \leq r_{1}-k, \\
+\infty & \text { otherwise }\end{cases}\right.
$$

Let $s$ and $t$ be distinct elements not belonging to $V_{1} \cup V_{2}$, and define a function $h$ on $\mathbf{Z}^{V_{1} \cup\{s\} \cup V_{2} \cup\{t\}}$ by the disjoint sum of $f_{2}, g_{2}$ with the simultaneous coordinate inversion and $f_{1}, g_{1}$, i.e.,

$$
h\left(x_{1}, p, x_{2}, q\right):=\left(f_{1}\left(-x_{1}\right)+g_{1}(-p)\right)+\left(f_{2}\left(x_{2}\right)+g_{2}(q)\right)
$$

for each $x_{1} \in \mathbf{Z}^{V_{1}}, x_{2} \in \mathbf{Z}^{V_{2}}$, and $(p, q) \in \mathbf{Z}^{\{s, t\}}$. It then follows that $h$ is an $\mathrm{M}^{\natural}$-convex function. Indeed, $g_{1}$ and $g_{2}$ are clearly $\mathrm{M}^{\natural}$-convex, the simultaneous coordinate inversion keeps the $\mathrm{M}^{\natural}$ convexity (see, e.g., [32, Theorem $6.13(2)]$ ), and the disjoint sum of two $\mathrm{M}^{\natural}$-convex functions is 
also $\mathrm{M}^{\natural}$-convex. We then construct a directed bipartite graph $G=\left(V_{1} \cup\{s\}, V_{2} \cup\{t\} ; A\right)$ endowed with a weight function $\hat{w}: A \rightarrow \mathbf{R}$ defined by

$$
\begin{aligned}
& A:=\left\{\left(v^{1}, v^{2}\right) \mid v \in V\right\} \cup\left\{\left(v^{1}, t\right) \mid v \in V\right\} \cup\left\{\left(s, v^{2}\right) \mid v \in V\right\}, \\
& \hat{w}(a):=\left\{\begin{array}{ll}
w(v) & \text { if } a=\left(v^{1}, v^{2}\right), \\
0 & \text { otherwise }
\end{array} \quad(a \in A) .\right.
\end{aligned}
$$

We now obtain the following instance of the $\mathrm{M}^{\natural}$-convex submodular flow problem:

$$
\begin{array}{lll}
\text { Minimize } & h(\partial \xi)+\sum_{a \in A} \hat{w}(a) \xi(a) & \\
\text { subject to } & \xi(a) \geq 0 \quad(a \in A) .
\end{array}
$$

The following lemma shows that $\left(\mathrm{M}_{\geq k}(w)\right)$ with $w \leq 0$ is reduced to the problem (3), and thus establishes its tractability.

Lemma 14. Problem $\left(\mathrm{M}_{\geq k}(w)\right)$ with $w \leq 0$ is equivalent to the problem (3).

Proof. Given a feasible solution $\left(x_{1}, x_{2}\right)$ of $\left(\mathrm{M}_{\geq k}(w)\right)$, construct a feasible solution $\xi$ of $(3)$ by

$$
\xi(a):= \begin{cases}\min \left\{x_{1}(v), x_{2}(v)\right\} & \text { if } a=\left(v^{1}, v^{2}\right), \\ \max \left\{0, x_{1}(v)-x_{2}(v)\right\} & \text { if } a=\left(v^{1}, t\right), \\ \max \left\{0, x_{2}(v)-x_{1}(v)\right\} & \text { if } a=\left(s, v^{2}\right) .\end{cases}
$$

Then it is not difficult to see that $\left(x_{1}, x_{2}\right)$ in $\left(\mathrm{M}_{\geq k}(w)\right)$ and $\xi$ in (3) have the same objective values.

Conversely, take any feasible solution $\xi$ for (3). If $\xi\left(v^{1}, t\right)=0$ or $\xi\left(s, v^{2}\right)=0$ holds for every $v \in V$, then we can straightforwardly construct a feasible solution $\left(x_{1}, x_{2}\right)$ satisfying (4). In this case, the objective value of $\left(x_{1}, x_{2}\right)$ in $\left(\mathrm{M}_{\geq k}(w)\right)$ and that of $\xi$ in (3) are the same. Suppose that there is $v \in V$ with $\xi\left(v^{1}, t\right)>0$ and $\xi\left(s, v^{\overline{2}}\right)>0$. Then define a new feasible solution $\xi^{\prime}$ of (3) by

$$
\xi^{\prime}(a):= \begin{cases}\xi(a)+\min \left\{\xi\left(v^{1}, t\right), \xi\left(s, v^{2}\right)\right\} & \text { if } a=\left(v^{1}, v^{2}\right), \\ \xi(a)-\min \left\{\xi\left(v^{1}, t\right), \xi\left(s, v^{2}\right)\right\} & \text { if } a=\left(v^{1}, t\right) \text { or } a=\left(s, v^{2}\right) .\end{cases}
$$

Clearly $\partial \xi=\partial \xi^{\prime}$ holds. It follows from $w \leq 0$ that the objective value for $\xi^{\prime}$ is at most that for $\xi$. It also follows that $\xi^{\prime}\left(v^{1}, t\right)=0$ or $\xi^{\prime}\left(s, v^{2}\right)=0$ holds for each $v \in V$. We can thus construct a feasible solution $\left(x_{1}, x_{2}\right)$ satisfying (4), attaining the desired objective value. Therefore we conclude that $\left(\mathrm{M}_{\geq k}(w)\right)$ with $w \leq 0$ and the problem (3) are equivalent.

\section{The NP-hardness of $\left(\mathrm{V}^{n}(w)\right)$ and $\left(\mathrm{M}_{\geq k}(w)\right)$}

In Sections 4 and 5, we have shown the tractability of $\left(\mathrm{V}^{n}(w)\right)$ with $w \geq 0$, and that of $\left(\mathrm{M}_{\geq k}(w)\right)$ with $w \leq 0$. This section is devoted to showing that they are NP-hard in general.

The NP-hardness of $\left(\mathrm{V}^{n}(w)\right)$. We prove that $\left(\mathrm{V}^{n}(w)\right)$ can formulate the problem of finding a maximum common independent set for three matroids, which is NP-hard. Given three matroids $M_{1}, M_{2}, M_{3}$ on the same ground set $V$ with the base families $\mathcal{B}_{1}, \mathcal{B}_{2}, \mathcal{B}_{3}$, respectively, construct an instance of $\left(\mathrm{V}^{n}(w)\right)$ as follows. Let $\delta_{\mathcal{B}_{i}}$ be the indicator function of $\mathcal{B}_{i}$ for $i=1,2,3$, and define a weight function $w: V \rightarrow \mathbf{R}$ by $w(v):=-1$ for each $v \in V$. Clearly, $\delta_{\mathcal{B}_{i}}$ is a valuated matroid for each $i=1,2,3$. Thus, $\delta_{\mathcal{B}_{i}}(i=1,2,3)$ and $w$ define an instance of $\left(\mathrm{V}^{n}(w)\right)$ with $n=3$. It is straightforward to see that this instance of $\left(\mathrm{V}^{n}(w)\right)$ is equivalent to the problem of finding a maximum comment independent set of $M_{1}, M_{2}, M_{3}$, as required. 
The NP-hardness of $\left(\mathrm{M}_{\geq k}(w)\right)$. Lendl et al. [24] proved that the following problem $\left(\mathrm{P}_{=0}\right)$ is NP-hard:

$$
\begin{array}{lll} 
& \text { Minimize } & w_{1}\left(x_{1}\right)+w_{2}\left(x_{2}\right) \\
\text { subject to } & x_{i} \in B_{i} \\
& \sum_{v \in V} \min \left\{x_{1}(v), x_{2}(v)\right\}=0,
\end{array} \quad(i=1,2),
$$

where $B_{1}, B_{2} \subseteq \mathbf{Z}^{V}$ are the base polytopes of some polymatroids on the ground set $V$, and $w_{1}$ and $w_{2}$ are linear functions on $\mathbf{Z}^{V}$. Here we prove that $\left(\mathrm{P}_{=0}\right)$ can be reduced to $\left(\mathrm{M}_{\geq k}(w)\right)$.

Given an instance of $\left(\mathrm{P}_{=0}\right)$, construct an instance of $\left(\mathrm{M}_{\geq k}(w)\right)$ in the following way. For $i=1,2$, define a function $f_{i}: \mathbf{Z}^{V} \rightarrow \mathbf{R} \cup\{+\infty\}$ by

$$
f_{i}\left(x_{i}\right):= \begin{cases}w_{i}\left(x_{i}\right) & \text { if } x_{i} \in B_{i} \\ +\infty & \text { otherwise. }\end{cases}
$$

It is not difficult to see that $f_{1}$ and $f_{2}$ are M-convex functions. Set $k=0$ and $w$ sufficiently large, e.g., $w(v)>\max _{x_{1} \in B_{1}} f_{1}\left(x_{1}\right)+\max _{x_{2} \in B_{2}} f_{2}\left(x_{2}\right)$ for each $v \in V$. Denote an instance of $\left(\mathrm{M}_{\geq k}(w)\right)$ defined by these $f_{1}, f_{2}, k, w$ by (I).

Then, an optimal solution for the given instance of $\left(\mathrm{P}_{=0}\right)$ can be obtained from that of (I). If (I) is infeasible, then clearly the instance of $\left(\mathrm{P}_{=0}\right)$ is infeasible. Suppose that (I) has an optimal solution $\left(x_{1}^{*}, x_{2}^{*}\right)$ with bounded objective value. If $\sum_{v \in V} \min \left\{x_{1}^{*}(v), x_{2}^{*}(v)\right\}>0$, it follows from the construction of $w$ that there is no $\left(x_{1}, x_{2}\right)$ satisfying that $x_{1} \in B_{1}, x_{2} \in B_{2}$, and $\sum_{v \in V} \min \left\{x_{1}^{*}(v), x_{2}^{*}(v)\right\}=0$, which implies that the given instance of $\left(\mathrm{P}_{=0}\right)$ is infeasible. If $\sum_{v \in V} \min \left\{x_{1}^{*}(v), x_{2}^{*}(v)\right\}=0$, then it is straightforward to see that $\left(x_{1}^{*}, x_{2}^{*}\right)$ is also an optimal solution for the instance of $\left(\mathrm{M}_{\geq k}(w)\right)$.

\section{Applications}

In this section, we present applications of our generalized problems in the recoverable robust matroid basis problem, combinatorial optimization problems with interaction costs, and matroid congestion games.

\subsection{Recoverable robust matroid basis problem}

In the recoverable robust matroid basis problem [3], we are given a matroid $(V, \mathcal{B})$ with ground set $V$ and base family $\mathcal{B} \subseteq 2^{V}$, a weight function $w_{1}$ on $V$, a family $\mathcal{W}$ of weight functions on $V$, and a nonnegative integer $k$. The recoverable robust matroid basis problem is described as the following minimization problem with variable $X_{1} \in \mathcal{B}$ :

$$
\begin{array}{ll}
\text { Minimize } & w_{1}\left(X_{1}\right)+\max _{w_{2} \in \mathcal{W}}\left\{\min _{X_{2} \in \mathcal{B},\left|X_{1} \cap X_{2}\right| \geq k} w_{2}\left(X_{2}\right)\right\} \\
\text { subject to } & X_{1} \in \mathcal{B} .
\end{array}
$$

This problem simulates the following situation. The family $\mathcal{W}$ represents the uncertainty of cost functions. The actual cost function $w_{2} \in \mathcal{W}$ is revealed after choosing a basis $X_{1} \in \mathcal{B}$, which costs $w_{1}\left(X_{1}\right)$. In the recovery phase, we rechoose a basis $X_{2} \in \mathcal{B}$ that is not much different from the first basis $X_{1}$, i.e., $\left|X_{1} \cap X_{2}\right| \geq k$, which requires the additional cost $w_{2}\left(X_{2}\right)$. The objective is to minimize the worst-case total cost $w_{1}\left(X_{1}\right)+w_{2}\left(X_{2}\right)$.

It is known [19] that the recoverable robust matroid basis problem is NP-hard even when $|\mathcal{W}|$ is constant and $\mathcal{B}$ is a base family of a graphic matroid. Lendl et al. [24] observed that the 
recoverable robust matroid basis problem can be reduced to $\left(\mathrm{W}_{\geq k}\right)$ if the uncertainty set $\mathcal{W}$ has the interval uncertainty representation:

$$
\mathcal{W}=\{w: V \rightarrow \mathbf{R} \mid \underline{w}(v) \leq w(v) \leq \bar{w}(v) \text { for each } v \in V\} .
$$

Indeed, in this case, (5) can be described in the form of $\left(\mathrm{W}_{\geq k}\right)$ :

$$
\begin{array}{ll}
\text { Minimize } & w_{1}\left(X_{1}\right)+\bar{w}\left(X_{2}\right) \\
\text { subject to } & X_{1}, X_{2} \in \mathcal{B} \\
& \left|X_{1} \cap X_{2}\right| \geq k
\end{array}
$$

Our result naturally gives its nonlinear and polymatroidal generalization. Let $f_{1}$ and $f$ be M-convex functions on $\mathbf{Z}^{V}$ with $\operatorname{dom} f_{1}, \operatorname{dom} f \subseteq \mathbf{Z}_{+}^{V}$. Define a family $\mathcal{W}$ of M-convex functions by

$$
\mathcal{W}=\left\{f+w \mid w: \text { linear function on } \mathbf{Z}^{V} \text { with } \underline{w} \leq w \leq \bar{w}\right\}
$$

where $\underline{w}$ and $\bar{w}$ are linear functions on $\mathbf{Z}^{V}$. Now, consider the following problem:

$$
\text { Minimize } f_{1}\left(x_{1}\right)+\max _{f_{2} \in \mathcal{W}}\left\{\min \left\{f_{2}\left(x_{2}\right) \mid \sum_{v \in V} \min \left\{x_{1}(v), x_{2}(v)\right\} \geq k\right\}\right\} .
$$

It then follows that (7) amounts to

$$
\begin{array}{ll}
\text { Minimize } & f_{1}\left(x_{1}\right)+\left(f_{2}+\bar{w}\right)\left(x_{2}\right) \\
\text { subject to } & \sum_{v \in V} \min \left\{x_{1}(v), x_{2}(v)\right\} \geq k .
\end{array}
$$

Since $f_{2}+\bar{w}$ is M-convex, this is a special case of $\left(\mathrm{M}_{\geq k}(w)\right)$ with $w \leq 0$, and thus can be solved in weakly polynomial time. In particular, if the objective function is defined on $2^{V}$, or equivalently, $f_{1}$ and $f_{2}$ are valuated matroids, then (7) is equivalent to $\left(\mathrm{V}_{\geq k}\right)$, and can be solved in strongly polynomial time.

Theorem 15. The problem (7) can be solved in weakly polynomial time when the uncertainty set $\mathcal{W}$ is in the form of (6). In addition, if the objective function is defined on $2^{V}$, then it can be solved in strongly polynomial time.

Let us also mention the following variant of the recoverable robust matroid basis problem discussed in Lendl et al. [24]. Here, $w_{1}$ and $w_{2}$ are weight functions on $V, \mathcal{B}_{1}$ and $\mathcal{B}_{2}$ are the base families of matroids on $V$, and $c: \mathbf{Z}_{+} \rightarrow \mathbf{R} \cup\{+\infty\}$ is a univariate function which is not necessarily linear.

$$
\begin{array}{lll}
\left(\mathrm{W}_{c}\right) & \begin{array}{l}
\text { Minimize } \\
\text { subject to }
\end{array} \quad w_{1}\left(X_{1}\right)+w_{2}\left(X_{2}\right)+c\left(\left|X_{1} \cap X_{2}\right|\right) & \\
& \text { Sub } &
\end{array} \quad(i=1,2) .
$$

They showed that $\left(\mathrm{W}_{c}\right)$ can be solved in strongly polynomial time by reducing it to $\left(\mathrm{W}_{=k}\right)$. Indeed, for each $k=0,1, \ldots,|V|$, let $\left(X_{1}^{k}, X_{2}^{k}\right)$ be an optimal solution of $\left(\mathrm{W}_{=k}\right)$. Then we have that $\min _{k=0,1, \ldots,|V|}\left\{w_{1}\left(X_{1}^{k}\right)+w_{2}\left(X_{2}^{k}\right)+c(k)\right\}$ is an optimal solution of $\left(\mathrm{W}_{c}\right)$. We remark that the original form in [24] includes a penalty $C\left(\left|X_{1} \triangle X_{2}\right|\right)$ in place of $c\left(\left|X_{1} \cap X_{2}\right|\right)$, where $C$ is also a univariate function. It follows that $c$ and $C$ have a one-to-one correspondence defined by a certain transformation.

Here we generalize the weight functions $w_{1}$ and $w_{2}$ in $\left(\mathrm{W}_{c}\right)$ to valuated matroids $\omega_{1}$ and $\omega_{2}$ on $2^{V}$ :

$$
\left(\mathrm{V}_{c}\right) \quad \text { Minimize } \quad \omega_{1}\left(X_{1}\right)+\omega_{2}\left(X_{2}\right)+c\left(\left|X_{1} \cap X_{2}\right|\right) .
$$


By the same argument for $\left(\mathrm{W}_{c}\right)$, we can solve $\left(\mathrm{V}_{c}\right)$ in strongly polynomial time via $\left(\mathrm{V}_{=k}\right)$.

Besides $\left(\mathrm{V}_{=k}\right)$, the problem $\left(\mathrm{V}_{c}\right)$ has some similarity to other problems discussed so far. It first looks similar to $\left(\mathrm{V}^{n}(w)\right)$ with $n=2$, but in fact they are different in the sense that the additional costs $c\left(\left|X_{1} \cap X_{2}\right|\right)$ and $w\left(X_{1} \cap X_{2}\right)$ cannot represent each other: they just coincide in the cases where $c$ is a linear function and $w(v)$ is identical for every $v \in V$.

This observation, however, leads to the following generalization of $\left(\mathrm{V}_{c}\right)$. That is, we can generalize $\left(\mathrm{V}_{c}\right)$ to have $n$ valuated matroids instead of the two weight functions $w_{1}$ and $w_{2}$, and can solve it in strongly polynomial time by our solution to $\left(\mathrm{V}^{n}(w)\right)$ if $c$ is linear and nonnegative. Similarly, we can generalize $\left(\mathrm{V}_{c}\right)$ to have two M-convex functions on $\mathbf{Z}_{+}^{V}$, and can solve it in weakly polynomial time via $\left(\mathrm{M}_{\geq k}(w)\right)$ if $c$ is linear and nonpositive.

\subsection{Combinatorial optimization problem with interaction costs}

Lendl, Ćustić, and Punnen [23] introduced a framework of combinatorial optimization with interaction costs $(C O P I C)$, which is described as follows. For two sets $V_{1}$ and $V_{2}$, we are given cost functions $w_{1}: V_{1} \rightarrow \mathbf{R}$ and $w_{2}: V_{2} \rightarrow \mathbf{R}$, as well as interaction costs $q: V_{1} \times V_{2} \rightarrow \mathbf{R}$. The objective is to find a pair of feasible sets $X_{1} \subseteq V_{1}$ and $X_{2} \subseteq V_{2}$ minimizing

$$
\sum_{u \in X_{1}} w_{1}(u)+\sum_{v \in X_{2}} w_{2}(v)+\sum_{u \in X_{1}} \sum_{v \in X_{2}} q(u, v)
$$

We focus on the diagonal COPIC, where $V_{1}$ and $V_{2}$ are identical and $q(u, v)=0$ if $u \neq v$. We further assume that the feasible sets are the base families of matroids. That is, the problem is formulated by two matroids $\left(V, \mathcal{B}_{1}\right)$ and $\left(V, \mathcal{B}_{2}\right)$ and modular cost functions $w_{1}, w_{2}, q: 2^{V} \rightarrow \mathbf{R}$ in the following way:

$$
\begin{array}{lll}
\text { Minimize } & w_{1}\left(X_{1}\right)+w_{2}\left(X_{2}\right)+q\left(X_{1} \cap X_{2}\right) & \\
\text { subject to } & X_{i} \in \mathcal{B}_{i} & (i=1,2) .
\end{array}
$$

The problem (8) appears in the context of the 2-min-max-min robustness [2] defined as follows:

$$
\min _{X_{1}, X_{2} \in \mathcal{B}} \max _{w \in \mathcal{W}} \min \left\{w\left(X_{1}\right), w\left(X_{2}\right)\right\}
$$

where $\mathcal{B} \subseteq 2^{V}$ is the base family of a matroid and $\mathcal{W}$ is a family of cost functions on $V$. Chassein and Goerigk [4] showed that, if $\mathcal{W}$ is of the form

$$
\mathcal{W}=\left\{w: V \rightarrow \mathbf{R}_{+} \mid \underline{w}(v) \leq w(v) \leq \bar{w}(v)(v \in V), \sum_{v \in V} \frac{w(v)-\underline{w}(v)}{\bar{w}(v)-\underline{w}(v)} \leq C\right\}
$$

for some $C \in \mathbf{R}_{+}$, then the 2-min-max-min robustness can be reduced to $O\left(|V|^{3}\right)$ many problems in the form (8), where $\mathcal{B}_{1}=\mathcal{B}_{2}, w_{1}$ and $w_{2}$ are nonpositive weight functions, and $q$ is a nonnegative weight function on $V$.

Chassein and Goerigk [4] proved that the above special case of the problem (8) can be solved in polynomial time via the ellipsoid method. Other previous work on the problem (8) includes the following. If $w_{1}$ and $w_{2}$ are identically zero and $q \geq 0$, then the problem (8) amounts to finding a socially optimal state in a two-player matroid congestion game, and thus can be solved in polynomial time [1]. Lendl et al. [23] extended the solvability to the case where the interaction cost $q$ may be arbitrary.

Now we can discuss another direction of generalization: the costs $w_{1}$ and $w_{2}$ are valuated matroids. This is a special case of $\left(\mathrm{V}^{n}(w)\right)$ and $\left(\mathrm{M}_{\geq k}(w)\right)$, and thus can be solved in polynomial time when $q \geq 0$ or $q \leq 0$. 
Theorem 16. The problem (8) can be solved in strongly polynomial time if $w_{1}, w_{2}: 2^{V} \rightarrow \mathbf{R}$ are valuated matroids, and $q \geq 0$ or $q \leq 0$.

As mentioned above, Chassein-Goerigk's algorithm for the problem (8) corresponding to the 2-min-max-min robustness is based on the ellipsoid method and hence not combinatorial. In contrast, our algorithm provides a combinatorial algorithm for this case of the problem (8). More generally, our result leads to the first combinatorial algorithm for the 2-min-max-min robustness (9) with $\mathcal{W}$ in the form (10).

\subsection{Socially optimal states in valuated matroid congestion games}

We finally present an application of $\left(\mathrm{V}^{n}(w)\right)$ in congestion games [37], a class of noncooperative games in game theory. A congestion game is represented by a tuple $\left(N, V,\left(\mathcal{B}_{i}\right)_{i \in N},\left(c_{v}\right)_{v \in V}\right)$, where $N=\{1,2, \ldots, n\}$ is a set of players, $V$ is a set of resources, $\mathcal{B}_{i} \subseteq 2^{V}$ is the set of strategies of a player $i \in N$, and $c_{v}: \mathbf{Z}_{+} \rightarrow \mathbf{R}_{+}$is a nondecreasing cost function associated with a resource $v \in V$. A state $\mathcal{X}=\left(X_{1}, X_{2}, \ldots, X_{n}\right)$ is a collection of strategies of all players, i.e., $X_{i} \in \mathcal{B}_{i}$ for each $i \in N$. For a state $\mathcal{X}=\left(X_{1}, X_{2}, \ldots, X_{n}\right)$, let $x^{(v)}(\mathcal{X})$ denote the number of players using $v$, i.e., $x^{(v)}(\mathcal{X})=\left|\left\{i \in N \mid v \in X_{i}\right\}\right|$. If $\mathcal{X}$ is clear from the context, $x^{(v)}(\mathcal{X})$ is abbreviated as $x^{(v)}$. In a state $\mathcal{X}$, every player using a resource $v \in V$ should pay $c_{v}\left(x^{(v)}\right)$ to use $v$, and thus the total cost paid by a player $i \in N$ is $\sum_{v \in X_{i}} c_{v}\left(x^{(v)}\right)$. In a player specific-cost model, the cost paid by a player $i \in N$ for using $v \in V$ is represented by a function $c_{i, v}: \mathbf{Z}_{+} \rightarrow \mathbf{R}_{+}$, which may vary with each player.

The importance of congestion games is appreciated through the fact that the class of congestion games coincides with that of potential games. Rosenthal [37] proved that every congestion game is a potential game, and conversely, Monderer and Shapley [25] proved that every potential game is represented by a congestion game with the same potential function.

Here we show that, in a certain generalized model of matroid congestion games with playerspecific costs, computing a socially optimal state reduces to (a variant of) $\left(\mathrm{V}^{n}(w)\right)$. A state $\mathcal{X}^{*}=\left(X_{1}^{*}, X_{2}^{*}, \ldots, X_{n}^{*}\right)$ is called socially optimal if the sum of the costs paid by all the players is minimum, i.e.,

$$
\sum_{i \in N} \sum_{v \in X_{i}^{*}} c_{v}\left(x^{(v)}\left(\mathcal{X}^{*}\right)\right) \leq \sum_{i \in N} \sum_{v \in X_{i}} c_{v}\left(x^{(v)}(\mathcal{X})\right)
$$

for any state $\mathcal{X}=\left(X_{1}, X_{2}, \ldots, X_{n}\right)$. In a matroid congestion game, the set $\mathcal{B}_{i} \subseteq 2^{V}$ of the strategies of each player $i \in N$ is the base family of a matroid on $V$. A socially optimal state in matroid congestion games can be computed in polynomial time if the cost functions are weakly convex [1, 41], while it is NP-hard for general nondecreasing cost functions [1]. A function $c: \mathbf{Z}_{+} \rightarrow \mathbf{R}$ is called weakly convex if $(x+1) \cdot c(x+1)-x \cdot c(x)$ is nondecreasing for each $x \in \mathbf{Z}_{+}$.

We consider the following generalized model of congestion games with player-specific costs. In a state $\mathcal{X}=\left(X_{1}, X_{2}, \ldots, X_{n}\right)$, the cost paid by a player $i \in N$ is

$$
\omega_{i}\left(X_{i}\right)+\sum_{v \in X_{i}} d_{v}\left(x^{(v)}\right)
$$

where $\omega_{i}: 2^{V} \rightarrow \mathbf{R}_{+}$is a monotone set function and $d_{v}: \mathbf{Z}_{+} \rightarrow \mathbf{R}_{+}$is a nondecreasing function for each $v \in V$. This model represents a situation where a player $i \in N$ should pay $\omega_{i}\left(X_{i}\right)$ regardless of the strategies of the other players, as well as $d_{v}\left(x^{(v)}\right)$ for every resource $v \in X_{i}$, which is an additional cost resulting from the congestion on $v$. It is clear that the standard model of congestion games is a special case where $\omega_{i}\left(X_{i}\right)=\sum_{v \in X_{i}} c_{v}(1)$ for every $i \in N$ and 
every $X_{i} \in \mathcal{B}_{i}$, and

$$
d_{v}(x)= \begin{cases}0 & (x=0) \\ c_{v}(x)-c_{v}(1) & (x \geq 1)\end{cases}
$$

In this model, the sum of the costs paid by all the players is equal to

$$
\sum_{i \in N} \omega_{i}\left(X_{i}\right)+\sum_{v \in V} x^{(v)} \cdot d_{v}\left(x^{(v)}\right) .
$$

The following lemma is straightforward to see.

Lemma 17. The following are equivalent.

- $c_{v}$ is weakly convex.

- $d_{v}$ is weakly convex.

- $x \cdot d_{v}$ is discrete convex.

It follows from Lemma 17 that, if $c_{v}$ (or $d_{v}$ ) is weakly convex, then the function $\sum_{v \in V} x_{v}$. $d_{v}\left(x^{(v)}\right)$ is laminar convex.

The solution for $\left(\mathrm{V}^{n}(w)\right)$, or the DCA perspective for $\left(\mathrm{V}^{n}(w)\right)$, provides a new insight on this model of cost functions in matroid congestion games. In addition to the weak convexity of $d_{v}(v \in V)$, this model allows us to introduce some convexity of the cost function $\omega_{i}$. Namely, we can assume that $\omega_{i}$ is a valuated matroid for every $i \in N$. Then, computing the optimal state, i.e., minimizing (12), is naturally viewed as valuated matroid intersection problem for the valuated matroid $\sum_{i \in N} \omega_{i}\left(X_{i}\right)$ and the laminar convex function $\sum_{v \in V} x_{v} \cdot d_{v}\left(x_{v}\right)$ as in $\left(\mathrm{V}^{n}(w)\right)$. Thus it can be done in polynomial time.

Theorem 18. In a matroid congestion game in which each player's cost is represented by (11), the socially optimal state can be computed in strongly polynomial time if $\omega_{i}$ is a valuated matroid for each player $i \in N$ and $d_{v}$ is weakly convex for each resource $v \in V$.

\section{Discussions}

In this paper, we have presented several types of minimization of the sum of valuated matroids (or M-convex functions) under intersection constraints. We here consider the following another natural generalization of $\left(\mathrm{V}_{=k}\right)$, where $\omega_{1}$ and $\omega_{2}$ are valuated matroids on $2^{V}, w$ is a weight function on $V$, and $k$ is a nonnegative integer:

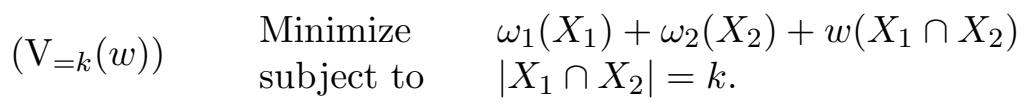

The problem $\left(\mathrm{V}_{=k}(w)\right)$ is similar to $\operatorname{VIAP}(k)$, but is essentially different. A problem that is similar to $\left(\mathrm{V}_{=k}(w)\right)$ and can be formulated by $\operatorname{VIAP}(k)$ is the following:

$$
\begin{array}{ll}
\text { Minimize } & \omega_{1}\left(X_{1}\right)+\omega_{2}\left(X_{2}\right)+w(F) \\
\text { subject to } & F \subseteq X_{1} \cap X_{2} \\
& |F|=k
\end{array}
$$

The difference between the problems $\left(\mathrm{V}_{=k}(w)\right)$ and (13) is that $\left|X_{1} \cap X_{2}\right|$ should be exactly equal to $k$ and all elements in $X_{1} \cap X_{2}$ affect the objective value in $\left(\mathrm{V}_{=k}(w)\right)$, whereas $\left|X_{1} \cap X_{2}\right|$ is just required to be at least $k$ and only $k$ elements in $X_{1} \cap X_{2}$ affect the objective value in (13). 
While $\operatorname{VIAP}(k)$ (and hence (13)) can be solved in polynomial time, the complexity of $\left(\mathrm{V}_{=k}(w)\right)$ is open even when the cardinality constraint $\left|X_{1} \cap X_{2}\right|=k$ is removed and $\omega_{1}$ and $\omega_{2}$ are modular functions on the base families of some matroids. For $\left(\mathrm{V}_{=k}(w)\right)$, only the following cases are known to be tractable:

- If $w$ is identically zero, then $\left(\mathrm{V}_{=k}(w)\right)$ is equivalent to $\left(\mathrm{V}_{=k}\right)$.

- If $w \geq 0$ and the cardinality constraint $\left|X_{1} \cap X_{2}\right|=k$ is removed, then $\left(\mathrm{V}_{=k}(w)\right)$ is a subclass of $\left(\mathrm{V}^{n}(w)\right)$ with $w \geq 0$.

- If $w \leq 0$ and $\left|X_{1} \cap X_{2}\right|=k$ is replaced by $\left|X_{1} \cap X_{2}\right| \geq k$, then $\left(\mathrm{V}_{=k}(w)\right)$ is a subclass of $\left(\mathrm{M}_{\geq k}(w)\right)$ with $w \leq 0$

- If $\left|X_{1} \cap X_{2}\right|=k$ is removed and $\omega_{1}$ and $\omega_{2}$ are the indicator functions of the base families of some matroids, then $\left(\mathrm{V}_{=k}(w)\right)$ has been dealt with Lendl et al. [23]; see Section 7.2.

Another possible direction of research would be to generalize our framework so that it includes computing the socially optimal state of polymatroid congestion games [12, 39], as we have done for matroid congestion games in Section 7.3. Polymatroid congestion games offer a model generalizing matroid congestion games where the usage of a resource by a player may not be binary, and its multiplicity can be represented by a nonnegative integer. In this model, the sum of the costs paid by all players for a resource $v$ may no longer be represented as $x^{(v)} \cdot d_{v}\left(x^{(v)}\right)$ as in (12), because the number of players using $v$ may not be equal to the multiplicity of the usage of $v$.

\section{Acknowledgements}

We thank András Frank and Kazuo Murota for careful reading and numerous helpful comments and Marc Goerigk for bibliographical information on [4]. We also thank the referees for helpful comments. The first author was supported by JSPS KAKENHI Grant Numbers JP17K00029, JP19J01302, 20K23323, 20H05795, Japan. The second author was supported by JSPS KAKENHI Grant Numbers JP16K16012, JP26280004, JP20K11699, Japan. This is a post-peerreview, pre-copyedit version of an article published in Mathematical Programming. The final authenticated version is available online at: https://doi.org/10.1007/s10107-021-01625-2.

\section{References}

[1] H. Ackermann, H. Röglin, and B. Vöcking. On the impact of combinatorial structure on congestion games. Journal of the ACM, 55(6):25:1-25:22, 2008.

[2] C. Buchheim and J. Kurtz. Min-max-min robust combinatorial optimization. Mathematical Programming, Series A, 163:1-23, 2017.

[3] C. Büsing. Recoverable Robustness in Combinatorial Optimization. Cuvillier Verlag, 2011.

[4] A. Chassein and M. Goerigk. On the complexity of min-max-min robustness with two alternatives and budgeted uncertainty. Discrete Applied Mathematics, to appear.

[5] A. W. M. Dress and W. Wenzel. Valuated matroids: A new look at the greedy algorithm. Applied Mathematics Letters, 3(2):33-35, 1990.

[6] A. W. M. Dress and W. Wenzel. Valuated matroids. Advances in Mathematics, 93:214-250, 1992. 
[7] J. Edmonds. Submodular functions, matroids, and certain polyhedra. In R. Guy, H. Hanani, N. Sauer, and J. Schönheim, editors, Combinatorial Structures and Their Applications, pages 69-87, New York, 1970. Gordon and Breach.

[8] J. Edmonds and R. Giles. A min-max relation for submodular functions on graphs. Annals of Discrete Mathematics, 1:185-204, 1977.

[9] A. Frank. A weighted matroid intersection algorithm. Journal of Algorithms, 2:328-336, 1981.

[10] M. L. Fredman and R. E. Tarjan. Fibonacci heaps and their uses in improved network optimization algorithms. Journal of the Association for Computing Machinery, 34:596-615, 1987.

[11] S. Fujishige. Submodular Functions and Optimization. Elsevier, Amsterdam, 2nd edition, 2005.

[12] T. Harks, M. Klimm, and B. Peis. Sensitivity analysis for convex separable optimization over integral polymatroids. SIAM Journal on Optimization, 28:2222-2245, 2018.

[13] R. Hassin. Minimum cost flow with set-constraints. Networks, 12(1):1-21, 1982.

[14] M. Hradovich, A. Kasperski, and P. Zielínski. Recoverable robust spanning tree problem under interval uncertainty representations. Journal of Combinatorial Optimization, $34(2): 554-573,2017$.

[15] M. Hradovich, A. Kasperski, and P. Zieliński. The recoverable robust spanning tree problem with interval costs is polynomially solvable. Optimization Letters, 11(1):17-30, 2017.

[16] M. Iri and N. Tomizawa. An algorithm for finding an optimal "independent assignment". Journal of the Operations Research Society of Japan, 19(1):32-57, 1976.

[17] S. Iwata, S. Moriguchi, and K. Murota. A capacity scaling algorithm for M-convex submodular flow. Mathematical Programming, Series A, 103:181-202, 2005.

[18] S. Iwata and M. Shigeno. Conjugate scaling algorithm for Fenchel-type duality in discrete convex optimization. SIAM Journal on Optimization, 13(1):204-211, 2003.

[19] A. Kasperski, A. Kurpisz, and P. Zieliíski. Recoverable robust combinatorial optimization problems. In Operations Research Proceedings 2012, pages 147-153, 2014.

[20] E. L. Lawler. Matroid intersection algorithms. Mathematical Programming, 9:31-56, 1975.

[21] E. L. Lawler and C. U. Martel. Computing maximal "polymatroidal" network flows. Mathematics of Operations Research, 7(3):334-347, 1982.

[22] E. L. Lawler and C. U. Martel. Flow network formulations of polymatroid optimization problems. Annals of Discrete Mathematics, 16:189-200, 1982.

[23] S. Lendl, A. Custić, and A. P. Punnen. Combinatorial optimization with interaction costs: Complexity and solvable cases. Discrete Optimization, 33:101-117, 2019.

[24] S. Lendl, B. Peis, and V. Timmermans. Matroid bases with cardinality constraints on the intersection. arXiv:1907.04741v2, 2019. 
[25] D. Monderer and L. S. Shapley. Potential games. Games and Economic Behavior, 14:124$143,1996$.

[26] K. Murota. Convexity and Steinitz's exchange property. Advances in Mathematics, 124:272$311,1996$.

[27] K. Murota. Valuated matroid intersection, I: optimality criteria. SIAM Journal on Discrete Mathematics, 9:545-561, 1996.

[28] K. Murota. Valuated matroid intersection, II: algorithms. SIAM Journal on Discrete Mathematics, 9:562-576, 1996.

[29] K. Murota. Discrete convex analysis. Mathematical Programming, 83:313-371, 1998.

[30] K. Murota. Submodular flow problem with a nonseparable cost function. Combinatorica, 19:87-109, 1999.

[31] K. Murota. Matrices and Matroids for Systems Analysis. Springer, Heidelberg, 2000.

[32] K. Murota. Discrete Convex Analysis. SIAM, Philadelphia, 2003.

[33] K. Murota. Recent developments in discrete convex analysis. In W. Cook, L. Lovász, and J. Vygen, editors, Research Trends in Combinatorial Optimization, chapter 11, pages 219-260. Springer, Heidelberg, 2009.

[34] K. Murota. Discrete convex analysis: A tool for economics and game theory. Journal of Mechanism and Institution Design, 1(1):151-273, 2016.

[35] K. Murota and A. Shioura. M-convex function on generalized polymatroid. Mathematics of Operations Research, 24(1):95-105, 1999.

[36] J. Oxley. Matroid Theory. Oxford University Press, 2nd edition, 2011.

[37] R. W. Rosenthal. A class of games possessing pure-strategy Nash equilibria. International Journal of Game Theory, 2:65-67, 1973.

[38] A. Schrijver. Combinatorial Optimization: Polyhedra and Efficiency. Springer, Heidelberg, 2003.

[39] K. Takazawa. Generalizations of weighted matroid congestion games: pure Nash equilibrium, sensitivity analysis, and discrete convex function. Journal of Combinatorial Optimization, $38: 1043-1065,2019$.

[40] L. Végh. Private communication. 2020.

[41] R. F. Werneck and J. C. Setubal. Finding minimum congestion spanning trees. ACM Journal of Experimental Algorithmics, 5:11, 2000.

\section{A Algorithm description and complexity analyses}

In this section, we describe the full behavior of the algorithm for $\left(\mathrm{V}_{\geq k}\right)$. We then analyse the time complexities of our algorithms for $\left(\mathrm{V}_{\geq k}\right)$ and $\left(\mathrm{V}_{=k}\right)$ to prove Theorem 4.

Suppose that, for some nonnegative integer $i<k$, we have at hand an optimal solution $\left(X_{1}, X_{2}\right)$ for $\left(\mathrm{V}_{\geq i}\right)$ and its optimality witness $\left(p_{1}, p_{2}, F\right)$; recall that the optimality condition has been described in Lemma 6 . We then find an optimal solution for $\left(\mathrm{V}_{\geq i+1}\right)$ and its optimality 
witness by utilizing an auxiliary digraph $\vec{G}=\left(V_{1} \cup V_{2} \cup\{s, t\}, A\right)$ endowed with a nonnegative arc-length function $\ell$ on $A$ defined from $\left(X_{1}, X_{2}\right)$ and $\left(p_{1}, p_{2}, F\right)$ as follows. Here $s$ and $t$ are new vertices, which play roles as a source vertex and sink vertex, respectively. The arc set $A$ is defined by

$$
A:=\vec{E} \cup \vec{F} \cup A_{1} \cup A_{2} \cup S \cup T
$$

where

$$
\begin{aligned}
\vec{E} & :=\left\{\left(v^{1}, v^{2}\right) \mid v \in V\right\}, \\
\vec{F} & :=\left\{\left(v^{2}, v^{1}\right) \mid v \in F\right\}, \\
A_{1} & :=\left\{\left(u^{1}, v^{1}\right) \mid u^{1} \in X_{1}, v^{1} \in V_{1} \backslash X_{1}, X_{1} \backslash\left\{u^{1}\right\} \cup\left\{v^{1}\right\} \in \operatorname{dom} \omega_{1}\right\}, \\
A_{2} & :=\left\{\left(v^{2}, u^{2}\right) \mid u^{2} \in X_{2}, v^{2} \in V_{2} \backslash X_{2}, X_{2} \backslash\left\{u^{2}\right\} \cup\left\{v^{2}\right\} \in \operatorname{dom} \omega_{2}\right\}, \\
S & :=\left\{\left(s, v^{1}\right) \mid v^{1} \in X_{1} \backslash X_{2}\right\}, \\
T & :=\left\{\left(v^{2}, t\right) \mid v^{2} \in X_{2} \backslash X_{1}\right\} .
\end{aligned}
$$

Define the arc-length function $\ell: A \rightarrow \mathbf{R}$ by

$$
\ell(a):= \begin{cases}\left(\omega_{1}-p_{1}\right)\left(X_{1} \backslash\left\{u^{1}\right\} \cup\left\{v^{1}\right\}\right)-\left(\omega_{1}-p_{1}\right)\left(X_{1}\right) & \text { if } a=\left(u^{1}, v^{1}\right) \in A_{1}, \\ \left(\omega_{2}+p_{2}\right)\left(X_{2} \backslash\left\{u^{2}\right\} \cup\left\{v^{2}\right\}\right)-\left(\omega_{2}+p_{2}\right)\left(X_{2}\right) & \text { if } a=\left(v^{2}, u^{2}\right) \in A_{2}, \\ 0 & \text { if } a \in A \backslash\left(A_{1} \cup A_{2}\right) .\end{cases}
$$

We remark that $\ell$ is nonnegative since $X_{1}$ and $X_{2}$ are minimizers of $\omega_{1}-p_{1}$ and $\omega_{2}+p_{2}$, respectively.

The augmenting path algorithm for $\left(\mathrm{V}_{\geq k}\right)$ runs in the auxiliary digraph as follows:

Step 1: Let $X_{1}$ and $X_{2}$ be the minimizers of $\omega_{1}$ and $\omega_{2}$, respectively.

- If $\left|X_{1} \cap X_{2}\right| \geq k$, then output $\left(X_{1}, X_{2}\right)$ and stop.

- Otherwise, let $k^{\prime}:=\left|X_{1} \cap X_{2}\right|<k$ and let $p_{1}, p_{2}$ be potential functions defined by $p_{1}\left(v^{1}\right)=p_{2}\left(v^{2}\right)=0$ for all $v \in V$. Then $\left(X_{1}, X_{2}\right)$ is an optimal solution for $\left(\mathrm{V}_{\geq k^{\prime}}\right)$ with optimality witness $\left(p_{1}, p_{2}, X_{1} \cap X_{2}\right)$.

Step 2: While $i=\left|X_{1} \cap X_{2}\right|<k$, do the following.

Suppose here that $\left(X_{1}, X_{2}\right)$ is an optimal solution for $\left(\mathrm{V}_{\geq i}\right)$ with optimality witness $\left(p_{1}, p_{2}, X_{1} \cap X_{2}\right)$. Let $\vec{G}$ be the auxiliary digraph for $\left(X_{1}, X_{2}\right)$ with $\left(p_{1}, p_{2}, X_{1} \cap X_{2}\right)$.

- If there is no $s$ - $t$ path in $\vec{G}$, then output " $\left(\mathrm{V}_{\geq k}\right)$ is infeasible" and stop.

- Otherwise, find a shortest $s$ - $t$ path $P$ in $\vec{G}$ with respect to the arc length $\ell$ such that the number of edges is smallest among the shortest $s$ - $t$ paths. For $x \in V_{1} \cup V_{2} \cup\{t\}$, let $d(x)$ be the length of the shortest $s-x$ path with respect to $\ell$ in $\vec{G}$, where $d(x):=+\infty$ if there is no $s-x$ path. Update $X_{1}, X_{2}, p_{1}$, and $p_{2}$ by

$$
\begin{aligned}
X_{1} & \leftarrow X_{1} \backslash\left(P \cap X_{1}\right) \cup\left(P \cap\left(V_{1} \backslash X_{1}\right)\right), \\
X_{2} & \leftarrow X_{2} \backslash\left(P \cap X_{2}\right) \cup\left(P \cap\left(V_{2} \backslash X_{2}\right)\right), \\
p_{1}\left(v^{1}\right) & \leftarrow p_{1}\left(v^{1}\right)+\min \left\{d\left(v^{1}\right), d(t)\right\} \quad \text { for } v^{1} \in V_{1}, \\
p_{2}\left(v^{2}\right) & \leftarrow p_{2}\left(v^{2}\right)-\min \left\{d\left(v^{2}\right), d(t)\right\} \quad \text { for } v^{2} \in V_{2} .
\end{aligned}
$$

Then, the resulting $X_{1}, X_{2}, p_{1}$, and $p_{2}$ satisfy that $\left|X_{1} \cap X_{2}\right|=i+1$ and $\left(X_{1}, X_{2}\right)$ is an optimal solution for $\left(\mathrm{V}_{\geq i+1}\right)$ with optimality witness $\left(p_{1}, p_{2}, X_{1} \cap X_{2}\right)$. 
Step 3: Output $\left(X_{1}, X_{2}\right)$ and stop.

We are ready to prove Theorem 4 . We first see the time complexity of the algorithm for $\left(\mathrm{V}_{\geq k}\right)$. We can obtain minimizers of $\omega_{1}$ and $\omega_{2}$ in $O(|V| r \gamma)$ time [5], and hence Step 1 can be done in $O(|V| r \gamma)$ time. Consider each iteration in Step 2. We can construct the auxiliary graph $\vec{G}$ in $O(|V| r \gamma)$ time. Indeed, the number of edges in $\vec{G}$ is $O(|V| r):|\vec{E}|$ is $O(|V|),|\vec{F}|,|S|$, and $|T|$ are $O(|r|),\left|A_{1}\right|$ is $O\left(r_{1}\left(|V|-r_{1}\right)\right)=O(|V| r)$, and $\left|A_{2}\right|$ is $O\left(r_{2}\left(|V|-r_{2}\right)\right)=O(|V| r)$, where $r_{1}$ and $r_{2}$ are the ranks of $\omega_{1}$ and $\omega_{2}$, respectively. Furthermore the arc-length function $\ell$ can be computed in $O\left(\left(\left|A_{1}\right|+\left|A_{2}\right|\right) \gamma\right)=O(|V| r \gamma)$ time. Then we can compute the length $d(x)$ of the shortest $s-x$ path for each $x \in V_{1} \cup V_{2} \cup\{s\}$ in $O(|V| r+|V| \log |V|)$ time by using Dijkstra's algorithm with Fibonacci heaps [10] (see also [38, Section 7.4]). The update of $\left(X_{1}, X_{2}\right)$ and $\left(p_{1}, p_{2}\right)$ requires $O(|V|)$ time. Therefore each iteration in Step 2 takes $O(|V| r \gamma+|V| \log |V|)$ time. Since the number of iterations is at most $k$, Step 2 can be done in $O(|V| r k \gamma+|V| k \log |V|)$ time. Thus the running-time of the algorithm for $\left(\mathrm{V}_{\geq k}\right)$ is $O(|V| r k \gamma+|V| k \log |V|)$.

We then consider the time complexity of the algorithm for $\left(\mathrm{V}_{=k}\right)$. Let $X_{1}$ and $X_{2}$ be minimizers of $\omega_{1}$ and $\omega_{2}$, respectively, which can be obtained in $O(|V| r \gamma)$ time. If $\left|X_{1} \cap X_{2}\right| \leq k$, then the algorithm for $\left(\mathrm{V}_{=k}\right)$ is the same as that for $\left(\mathrm{V}_{\geq k}\right)$, and hence the running-time is $O(|V| r k \gamma+|V| k \log |V|)$. If $\left|X_{1} \cap X_{2}\right|>k$, we solve $\left(\mathrm{V}_{\geq r_{1}-k}\right)$ for $\omega_{1}$ and $\overline{\omega_{2}}$. By the same argument as for the algorithm for $\left(\mathrm{V}_{\geq k}\right)$, one can see that the running-time of $\left(\mathrm{V}_{\geq r_{1}-k}\right)$ is $O\left(|V| r\left(r_{1}-k\right) \gamma+|V|\left(r_{1}-k\right) \log |V|\right)$. Altogether, $\left(\mathrm{V}_{=k}\right)$ can be solved in $O\left(|V| r^{2} \gamma+|V| r \log |V|\right)$ time.

\section{B Anothor solution to $\left(\mathrm{V}_{=k}\right)$}

This section provides another solution to $\left(\mathrm{V}_{=k}\right)$, which is mentioned at the end of Section 3.1. We first solve $\left(\mathrm{V}_{\leq k}\right)$ and $\left(\mathrm{V}_{\geq k}\right)$. The former is a special case of $\left(\mathrm{V}_{\mathcal{I}}^{n}\right)$ in which $n=2$ and $\mathcal{I}$ is the independent set family of the uniform matroid with rank $k$, and can be solved in $O\left(|V| r^{2} \gamma+\right.$ $|V| r \log |V|)$ time by Theorem 9. The latter can also be solved in $O\left(|V| r^{2} \gamma+|V| r \log |V|\right)$ time by Theorem 4 (or the reduction to valuated matroid intersection). If the output optimal solution $\left(X_{1}, X_{2}\right)$ of $\left(\mathrm{V}_{\leq k}\right)$ satisfies $\left|X_{1} \cap X_{2}\right|=k$ or that of $\left(X_{1}^{\prime}, X_{2}^{\prime}\right)$ of $\left(\mathrm{V}_{\geq k}\right)$ satisfies $\left|X_{1}^{\prime} \cap X_{2}^{\prime}\right|=k$, then we are done.

Otherwise, we have that $X_{1}$ and $X_{1}^{\prime}$ are minimizers of $\omega_{1}$, and $X_{2}$ and $X_{2}^{\prime}$ are those of $\omega_{2}$. Indeed, suppose, to the contrary, that $X_{1}$ is not a minimizer of $\omega_{1}$. Then there are $u \in X_{1}$ and $v \in V \backslash X_{1}$ such that $\omega_{1}\left(X_{1} \backslash\{u\} \cup\{v\}\right)<\omega_{1}\left(X_{1}\right)$. By $\left|X_{1} \cap X_{2}\right|<k$, it follows that $\left|\left(X_{1} \backslash\{u\} \cup\{v\}\right) \cap X_{2}\right| \leq k$. Therefore, $\left(X_{1} \backslash\{u\} \cup\{v\}, X_{2}\right)$ is feasible and $\omega_{1}\left(X_{1} \backslash\{u\} \cup\right.$ $\{v\})+\omega_{2}\left(X_{2}\right)<\omega_{1}\left(X_{1}\right)+\omega_{2}\left(X_{2}\right)$, which contradicts the optimality of $\left(X_{1}, X_{2}\right)$. By the same argument, we can conclude that $X_{1}^{\prime}$ is also a minimizer of $\omega_{1}$ and $X_{2}, X_{2}^{\prime}$ are minimizers of $\omega_{2}$.

Let $p=\left|X_{1}^{\prime} \backslash X_{1}\right|$ and $q=\left|X_{2}^{\prime} \backslash X_{2}\right|$. Since the set of minimizers of a valuated matroid forms a base family of some matroid, there is a sequence $\left(X_{1}^{0}, X_{1}^{1}, \ldots, X_{1}^{p}\right)$ of minimizers of $\omega_{1}$ such that $X_{1}^{0}=X_{1}$ and $\left|X_{1}^{\prime} \backslash X_{1}^{i}\right|=p-i$ for each $i=0,1, \ldots, p$. Note that $\left|X_{1}^{i-1} \cap X_{2}\right|-1 \leq\left|X_{1}^{i} \cap X_{2}\right| \leq$ $\left|X_{1}^{i-1} \cap X_{2}\right|+1$. If $\left|X_{1}^{i} \cap X_{2}\right|=k$ holds for some $i \in[p]$, then output $\left(X_{1}^{i}, X_{2}\right)$. Otherwise, we have that $\left|X_{1}^{\prime} \cap X_{2}\right|<k$. Then we can similarly consider a sequence $\left(X_{2}^{0}, X_{2}^{1}, \ldots, X_{2}^{q}\right)$ of minimizers of $\omega_{2}$, where $X_{2}^{0}=X_{2}$ and $\left|X_{2}^{\prime} \backslash X_{2}^{j}\right|=q-j$ for each $j=0,1, \ldots, q$. It then follows from $\left|X_{1}^{\prime} \cap X_{2}\right|<k$ and $\left|X_{1}^{\prime} \cap X_{2}^{\prime}\right|>k$ that there is $X_{2}^{j}$ with $\left|X_{1}^{\prime} \cap X_{2}^{j}\right|=k$. We thus output $\left(X_{1}^{\prime}, X_{2}^{j}\right)$ as an optimal solution. 


\section{The algorithm for $\left(\mathrm{W}_{=k}\right)$ by Lendl et al.}

The primal-dual algorithm of Lendl et al. [24] is described as follows. Let $X_{1} \in \operatorname{argmin}_{X \in \mathcal{B}_{1}} w_{1}(X)$ and $X_{2} \in \operatorname{argmin}_{X \in \mathcal{B}_{2}} w_{2}(X)$, respectively.

Case $1\left(\left|X_{1} \cap X_{2}\right| \leq k\right)$ : Let $k^{\prime}:=\left|X_{1} \cap X_{2}\right|$ and let $q_{1}, q_{2}$ be potential functions defined by $q_{1}\left(v^{1}\right)=q_{2}\left(v^{2}\right)=0$ for all $v \in V$.

While $\left|X_{1} \cap X_{2}\right|<k$, do the following. Let $\vec{G}$ be the auxiliary graph for $\left(X_{1}, X_{2}\right)$ with its LPT optimality witness $\left(q_{1}, q_{2}\right)$.

- Suppose that there is a zero length $s$ - $t$ path in $\vec{G}$ with respective to $\ell$. Then let $P$ be a zero length $s$ - $t$ path with the smallest number of edges, and update $X_{1}$ and $X_{2}$ by

$$
\begin{aligned}
& X_{1} \leftarrow X_{1} \backslash\left(P \cap X_{1}\right) \cup\left(P \cap\left(V_{1} \backslash X_{1}\right)\right), \\
& X_{2} \leftarrow X_{2} \backslash\left(P \cap X_{2}\right) \cup\left(P \cap\left(V_{2} \backslash X_{2}\right)\right) .
\end{aligned}
$$

- Suppose that there is no zero length $s$ - $t$ path in $\vec{G}$. Let $R$ be the set of vertices reachable from $s$ with a zero length path, and $\delta:=\min \left\{\ell(a) \mid a \in A_{1} \cup A_{2}, \ell(a)>0\right\}$. If $\delta=+\infty$, then output " $\left(\mathrm{W}_{=k}\right)$ is infeasible." Otherwise update $q_{1}$ and $q_{2}$ by

$$
\begin{aligned}
& q_{1}\left(v^{1}\right) \leftarrow \begin{cases}q_{1}\left(v^{1}\right)+\delta & \text { if } v^{1} \in V_{1} \cap R, \\
q_{1}\left(v^{1}\right) & \text { if } v^{1} \in V_{1} \backslash R,\end{cases} \\
& q_{2}\left(v^{2}\right) \leftarrow \begin{cases}q_{2}\left(v^{2}\right) & \text { if } v^{2} \in V_{2} \cap R, \\
q_{2}\left(v^{2}\right)-\delta & \text { if } v^{2} \in V_{2} \backslash R .\end{cases}
\end{aligned}
$$

Case $2\left(\left|X_{1} \cap X_{2}\right|>k\right)$ : Let $r_{1}$ be the rank of $M_{1}=\left(V, \mathcal{B}_{1}\right)$, and let $\overline{w_{2}}:=-w_{2}$ and $\overline{M_{2}}:=$ $\left(V,\left\{V \backslash B \mid B \in \mathcal{B}_{2}\right\}\right)$. Then apply Case 1 to $\left(\mathrm{W}_{=r_{1}-k}\right)$ with $\left(w_{1}, \bar{w}_{2} ; M_{1}, \overline{M_{2}}\right)$.

We discuss the difference of this algorithm and our algorithm in the updating procedures. The algorithm of Lendl et al. only considers the zero length edges in the auxiliary digraph $\vec{G}$ in updating a solution or potential functions, while our algorithm considers all edges in $\vec{G}$ to find the shortest paths with respect to $\ell$. Furthermore, as mentioned in Section 3.2, in the algorithm of Lendl et al., the update phases of a solution and of potential functions are completely separated, while our algorithm simultaneously updates a solution and potential functions. That is, for one update of a solution, the algorithm of Lendl et al. requires at most $|V|$ updates of potential functions, while our algorithm requires only one update of potential functions.

The difference in the running-times of the algorithms follows from the above arguments. Here we remark that, in $\left(\mathrm{W}_{\geq k}\right)$, we can compute the edge length $\ell(a)$ of $a$ in constant time for each $a$. In the algorithm of Lendl et al., one update of a solution takes $O\left(|V|^{2} r\right)$ time, since each update of potential functions requires $O(|V| r)$ time. Hence the time complexity of their algorithm is $O\left(|V|^{2} r^{2}\right)$. On the other hand, our algorithm requires $O(|V| r+|V| \log |V|)$ time for one update of a solution. Thus, the running-time of our algorithm is $O\left(|V| r^{2}+|V| r \log |V|\right)$ time by Theorem 4 with $\gamma=O(1)$. 